\title{
Fuzzy number division and the multi-granularity phenomenon
}

\author{
A. PIEGAT* and M. PLUCIŃSKI \\ Faculty of Computer Science and Information Technology, \\ West Pomeranian University of Technology, 49 Żołnierska St., 71-210 Szczecin, Poland
}

\begin{abstract}
The paper presents difficulties connected with fuzzy and interval division. If operations such as fuzzy addition, subtraction and multiplication provide as a result one compact, multidimensional granule, then a result of the fuzzy division can consists of few separated granules. Such results are more difficult to use in next calculations. The paper shows that the number of solution granules can be higher than 2 and that in certain problems division does not occur explicitly. In certain problems, separation of particular solution granules can be considerable. The paper also shows how to realize the fuzzy division when its denominator contains zero. Most types of fuzzy arithmetics forbid such operation. However, the paper shows that it is possible. Multidimensional fuzzy RDM arithmetic and horizontal membership functions which facilitate detecting of solution granules are also described. The considered problems are visualized by examples.
\end{abstract}

Key words: granular computing, fuzzy arithmetic, fuzzy calculations, RDM fuzzy arithmetic, horizontal membership function.

\section{Introduction}

Fuzzy arithmetic (FA) [1-7] is an extension of the interval arithmetic (IA) $[4,8]$. It extends a calculation domain from standard intervals to fuzzy intervals or fuzzy numbers. Both kinds of arithmetic are very important for the uncertainty theory [9], granular computing [4], grey systems [10] and computing with words $[11,12]$. IA and FA are necessary for solving linear and nonlinear systems of equations with uncertain coefficients [4]. Such systems describe real economic, engineering, medical, environmental protection (and many other) problems [13-16]. FA is a basis for intuitionistic fuzzy arithmetic $[17,18]$. It is also applied in rough-set problems to increase the solution quality [19].

FA meets with considerable interest of scientists and has been developed for many years. Numerous FA methods have been elaborated, e.g. L-R fuzzy arithmetic [20, 21], FA based on discretized fuzzy numbers and on the extension principle of Zadeh [22], FA using decomposed fuzzy membership functions (standard IA with $\alpha$-cuts) [22], advanced FA based on transformation method [23], constrained FA [22] etc. An overview of FA methods can be found in [3]. Some of FA methods allow to find a solution of simpler problems analytically, but more complicated ones must be solved numerically. All the time, we observe the emergence of new FA methods, so the question arises: what is the reason of such situation? Are existing methods not sufficiently effective?

The motivation of this paper is to present a phenomenon which can occur during the division of fuzzy numbers, intervals and other granular models of uncertain data. This phenomenon consists in a multi-granular division result and it does not occur in a division of conventional crisp numbers. The multi-granu-

*e-mail: apiegat@wi.zut.edu.pl

Manuscript submitted 2016-01-26, revised 2016-07-08, 2016-10-26 and 2016-12-30, initially accepted for publication 2017-01-20, published in August 2017. larity phenomenon occurs always when the uncertain divisor contains zero. If the divisor does not contain zero, then the division result always consists of a single granule. Examination of the problem has shown that if the multi-granularity occurs then the result usually consists of 2 granules, which is intuitively understandable. However, the division result can sometimes consist of more granules, e.g. of 4. The next surprising observation is that the distance between component granules, which intuitively should be infinitely small, can sometimes be quite considerable.

The multi-granularity of division result has been observed thanks to the application of a new type of fuzzy arithmetic that was called multidimensional fuzzy RDM arithmetic (MD-F-RDM arithmetic). In papers on other existing types of fuzzy arithmetic, the multi-granularity phenomenon has not been investigated because of a simple reason: the division by the fuzzy divisor containing zero is not allowed. Moreover, mathematical properties of these arithmetic types do not allow for such analysis. MD-F-RDM, which is shortly presented in Section 2, allows for this kind of division.

The paper is organized as follows: Section 1 describes new, horizontal membership functions (MFs) that are the basis of MD-F-RDM arithmetic. Other fuzzy arithmetic types use conventional, vertical MFs. Horizontal MFs considerably facilitate fuzzy calculations. A characteristic feature of MD-F-RDM arithmetic is that its calculation result is not a typical fuzzy set, but a multidimensional fuzzy solution set (MDFS-set). Such a set has properties of a complete, algebraic solution set and gives MD-F-RDM arithmetic properties which other fuzzy arithmetic types do not have. These properties are mentioned in Section 3. An additional property that has not been described in this section is the restoration property, owing to which MD-F-RDM arithmetic is able to satisfy the forward-backward calculation test. In Section 4, various examples of multi-granularity have been described. Among them, 
examples of multi-granularity greater than 2. Observation of the multi-granularity was possible thanks to MD-F-RDM arithmetic, which uses in calculations not only borders of intervals but also their insides. In Section 4, a real problem in which the multi-granularity can occur is also presented. It is the known system-balance problem of Leontief in which an economic country-system is described by a set of linear equation. Since at least a part of system parameters is uncertain, its determinant is also uncertain and can contain zero. Hence, the fuzzy solution set can be multi-granular.

Further on, a short description of the best known and frequently used standard fuzzy arithmetic [24] based on standard interval arithmetic [8] will be presented.

Uncertain values can be modelled in various ways. A very popular form is an interval [8]. Its definition is as follows: closed interval denoted by $[a, b]$ is the set of real numbers given by (1).

$$
[a, b]=\{x \in \mathbb{R}: a \leq x \leq b\}
$$

Fuzzy arithmetic deals with fuzzy numbers (FN) and fuzzy intervals (FI) [24]. FN and FI are special fuzzy sets defined on the set of real numbers $\mathbb{R}$. Their purpose is to approximate real, not precisely known, values of variables.

Given a fuzzy set $A$ defined on $\mathbb{R}$ and a real number $\mu \in[0,1]$, the crisp set $A_{\mu}=\{x \in \mathbb{R}: \quad A(x) \geq \mu\}$ is called $\mu$-cut of $A$. The crisp set $S(A)=\{x \in \mathbb{R}: \quad A(x)>0\}$ is called the support of $A$. When $\max _{x \in \mathbb{R}} A(x)=1, A$ is called a normal fuzzy set.

A fuzzy interval $A$ is a normal fuzzy set on $\mathbb{R}$ whose $\mu$-cuts for all $\mu \in(0,1]$ are closed intervals of real numbers and whose support is bounded. When $A(x)=1$ for exactly one $x \in \mathbb{R}$, then this special fuzzy interval is called a fuzzy number. A convenient way of expressing any fuzzy interval $A$ is the canonical form:

$$
A(x)= \begin{cases}f_{L}(x) & \text { for } x \in[a, b) \\ 1 & \text { for } x \in[b, c] \\ f_{R} & \text { for } x \in(c, d] \\ 0 & \text { otherwise }\end{cases}
$$

where $x \in \mathbb{R}, f_{L}$ is a real-valued function that is increasing and right-continuous, $f_{R}$ is a real-valued function that is decreasing and left-continuous. $f_{L}$ and $f_{R}$ can be called left and right border of $A . a, b, c, d$ are real numbers such that $a \leq b \leq c \leq d$. When $b=c, A$ is a fuzzy number. When $f_{L}$ and $f_{R}$ are linear functions we obtain a special type of fuzzy intervals called trapezoidal fuzzy intervals, which are dominant in applications. Any trapezoidal fuzzy interval $A$ is fully characterized by the quadruple $(a, b, c, d)$ of real numbers occurring in the special canonical form (3) (see Fig. 1).

$$
A(x)= \begin{cases}(x-a) /(b-a) & \text { for } x \in[a, b), \\ 1 & \text { for } x \in[b, c] \\ (d-x) /(d-c) & \text { for } x \in(c, d], \\ 0 & \text { otherwise. }\end{cases}
$$

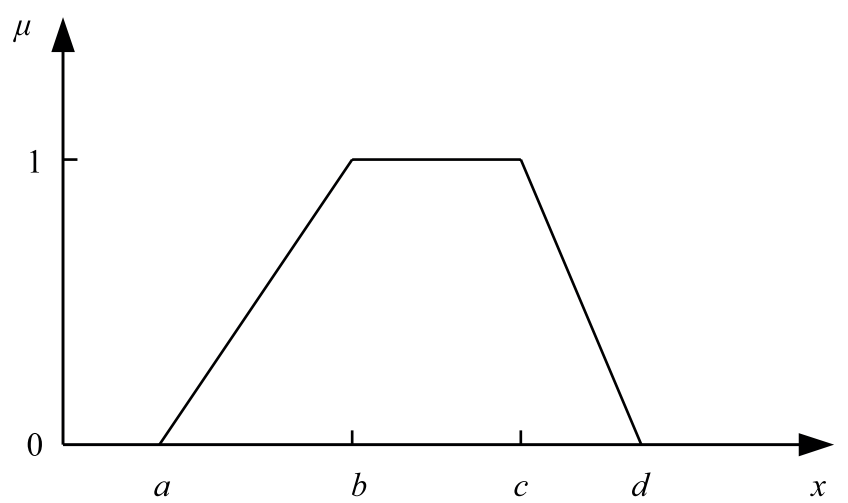

Fig. 1. Trapezoidal membership function

Let $A=(a, b, c, d)$ be used as a shorthand notation of trapezoidal fuzzy intervals. When $b=c$ in (3), $A$ is usually called a triangular fuzzy number.

The most widely used fuzzy arithmetic is called standard fuzzy arithmetic (SFA). In SFA, basic arithmetic operations on real numbers are extended to operations on fuzzy intervals. Two common ways of defining the extended operations are based on the $\mu$-cut representation of fuzzy intervals and on the extension principle of fuzzy set theory [20].

When the $\mu$-cut representation is applied, arithmetic operations on fuzzy intervals are defined in terms of arithmetic operations on closed intervals [8]. If we have two intervals $\left[a_{1}, a_{2}\right]$ and $\left[b_{1}, b_{2}\right]$ then:

$$
\left[a_{1}, a_{2}\right] \oplus\left[b_{1}, b_{2}\right]=\left[a_{1} \oplus b_{1}, a_{2} \oplus b_{2}\right]
$$

$$
\begin{aligned}
& {\left[a_{1}, a_{2}\right] \otimes\left[b_{1}, b_{2}\right]=} \\
& =\left[\min \left(a_{1} \otimes b_{1}, a_{1} \otimes b_{2}, a_{2} \otimes b_{1}, a_{2} \otimes b_{2}\right),\right. \\
& \left.\quad \max \left(a_{1} \otimes b_{1}, a_{1} \otimes b_{2}, a_{2} \otimes b_{1}, a_{2} \otimes b_{2}\right)\right],
\end{aligned}
$$

where: $\oplus \in\{+,-\}, \otimes \in\{\times, \div\}$ and $0 \notin\left[b_{1}, b_{2}\right]$ if $\otimes=\div$.

For fuzzy intervals $A$ and $B$ operations defined by (4) and (5) are realized for each $\mu$-cut, $\mu \in[0,1]$, which is the interval and can be denoted as $[\underline{a}(\mu), \bar{a}(\mu)]$ and $[\underline{b}(\mu), \bar{b}(\mu)]$.

SFA does not posses the inverse element of addition and multiplication with properties (6).

$$
X+(-X)=0, \quad X \cdot(1 / X)=1
$$

The sub-distributivity law and cancellation law for addition and multiplication do not hold in SFA. Therefore, application possibilities of SFA are limited.

Currently known methods are not perfect and can be improved. The multi-dimensional, relative distance measure fuzzy arithmetic (MD RDM-FA) is an alternative method of FA proposed and developed by Polish scientists from Szczecin [5-7, $11,13,18,25]$. Its basic concepts and the idea of horizontal membership functions (MF) were proposed by Piegat [25]. Further on, these concepts will be shortly presented. 
Figure 1 shows a trapezoidal MF (fuzzy interval). It can be noted that triangular or rectangular MFs are a special case of this function.

Vertical models of MFs are used in classic fuzzy systems. They express a vertical dependence $\mu=f(x)$ :

$$
\mu(x)= \begin{cases}(x-a) /(b-a) & \text { for } x \in[a, b), \\ 1 & \text { for } x \in[b, c], \\ (d-x) /(d-c) & \text { for } x \in(c, d], \\ 0 & \text { otherwise. }\end{cases}
$$

Formula (7) expresses a unique dependence of the 'vertical' variable $\mu$ from the 'horizontal' variable $x$. However, (7) is a model of MF borders only. Function (7) does not model an interior of MF. If such function is used in fuzzy calculations, only borders of MF (without its interior) are applied. It reduces the accuracy of achieved results. Vertical MFs also increase computational effort in fuzzy calculations. Hence, an idea of horizontal MFs was conceived.

However the question arises whether it is possible to create an inverse ('horizontal') model $x=f^{-1}(\mu)$. At first glance, it seems impossible because the dependence would not be unam-

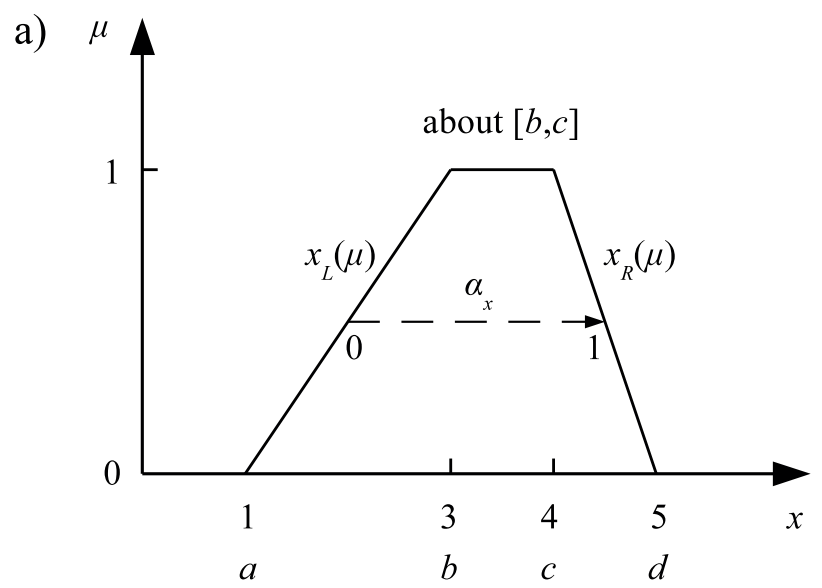

b)

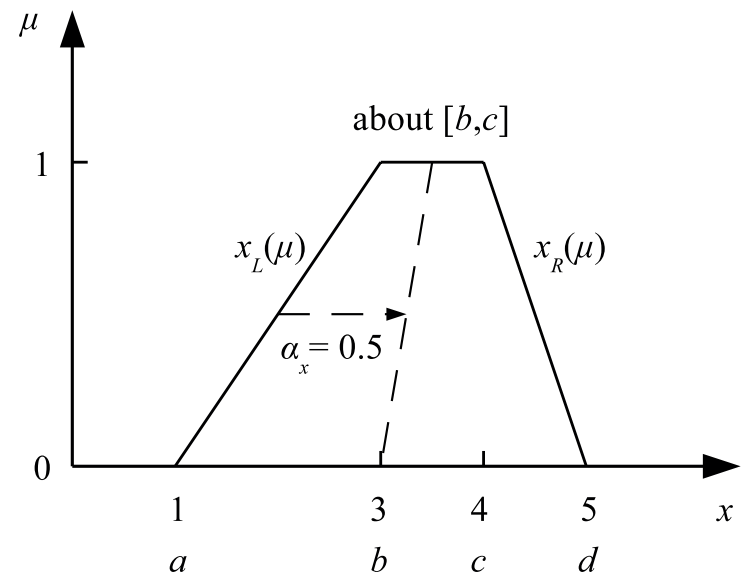

Fig. 2. Visualization of the horizontal approach to fuzzy membership functions biguous and it would not be a function then. However, it will be shown that such inverse model can be created.

Let us consider a horizontal cut of MF on the level $\mu$. Further on, this cut will be called $\mu$-cut (not $\alpha$-cut as usually), as in Fig. 2a. Variable $\alpha_{x}, \alpha_{x} \in[0,1]$, will be called RDM-variable (RDM - relative distance measure). It determines a relative distance of point $x^{*} \in\left[x_{L}(\mu), x_{R}(\mu)\right]$ from the origin of the local coordinate system (Fig. 2).

The RDM-variable $\alpha_{x}$ introduces the local Cartesian coordinate system into the interior of an interval. The left boundary $x_{L}(\mu)$ of MF and the right boundary $x_{R}(\mu)$ are expressed by (8).

$$
x_{L}=a+(b-a) \mu, \quad x_{R}=d-(d-c) \mu
$$

The RDM-variable $\alpha_{x}$ transforms the left boundary $x_{L}(\mu)$ into the right boundary $x_{R}(\mu)$. Contour line $x\left(\mu, \alpha_{x}\right)$ of constant $\alpha_{x}$ values is determined by (9).

$$
x\left(\mu, \alpha_{x}\right)=x_{L}+\left(x_{R}-x_{L}\right) \alpha_{x}, \quad \alpha_{x} \in[0,1]
$$

The contour line $x\left(\mu, \alpha_{x}\right)$ is set of points situated at an equal relative distance $\alpha_{x}$ from the left boundary of MF $x_{L}(\mu)$. A more precise form (10) of (9) can be called a horizontal MF.

$$
\begin{aligned}
x & =[a+(b-a) \mu] \\
& +[(d-a)-(d-c+b-a) \mu] \alpha_{x}, \quad \alpha_{x} \in[0,1]
\end{aligned}
$$

The horizontal MF $x=f\left(\mu, \alpha_{x}\right)$ is a function of two variables and exists in 3D-space (Fig. 3). It is unique.

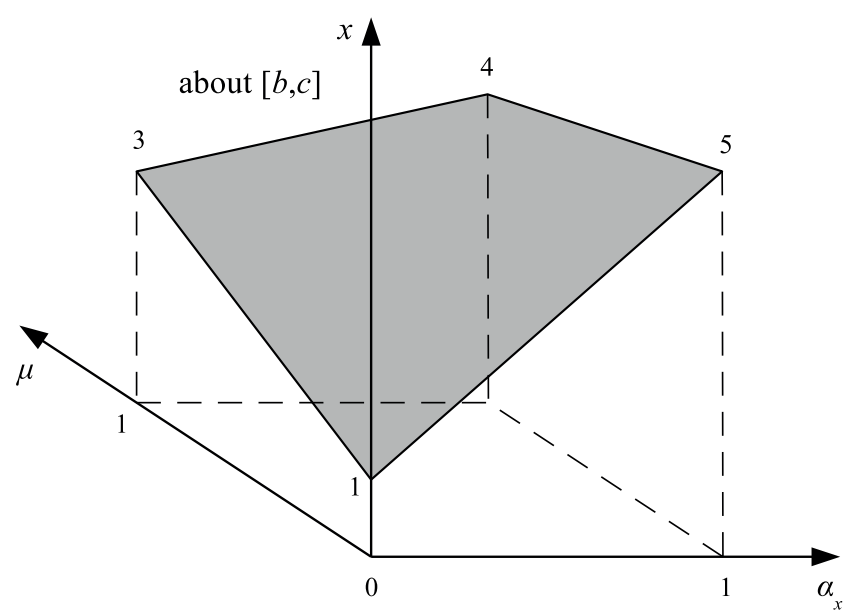

Fig. 3. Horizontal MF $x=(1+2 \mu)+(4-3 \mu) \alpha_{x}, \alpha_{x} \in[0,1]$, corresponding to vertical function shown in Fig. 2

The horizontal MF $x=f\left(\mu, \alpha_{x}\right)$ defines not a single value of the variable $x$, but a set of possible values of $x$ corresponding to a given $\mu$-cut level. It defines an information granule and hence it will be denoted as $x^{g r}$. Formula (10) describes the trapezoidal MF. However, it can be adapted to triangular MF by setting $b=c$ and to rectangular MF by setting $a=b$ and $c=d$. 
Boundaries of these functions are linear. To derive formulas for nonlinear boundaries, e.g. of Gauss type, formulas for the left and right boundary should be determined and used in (8).

\section{Operations of MD RDM fuzzy arithmetic}

Let $x^{g r}=f\left(\mu, \alpha_{x}\right)$ be a horizontal MF representing a fuzzy interval $X(11)$ and $y^{g r}=f\left(\mu, \alpha_{y}\right)$ be a horizontal MF representing a fuzzy interval $Y(12)$.

$$
\begin{aligned}
X: x^{g r} & =\left[a_{x}+\left(b_{x}-a_{x}\right) \mu\right] \\
& +\left[\left(d_{x}-a_{x}\right)-\left(d_{x}-c_{x}+b_{x}-a_{x}\right) \mu\right] \alpha_{x}, \\
& \mu, \alpha_{x} \in[0,1] \\
Y: y^{g r} & =\left[a_{y}+\left(b_{y}-a_{y}\right) \mu\right] \\
& +\left[\left(d_{y}-a_{y}\right)-\left(d_{y}-c_{y}+b_{y}-a_{y}\right) \mu\right] \alpha_{y}, \\
& \mu, \alpha_{y} \in[0,1]
\end{aligned}
$$

\section{Addition of two independent fuzzy intervals}

$$
\begin{aligned}
X+Y & =Z: x^{g r}\left(\mu, \alpha_{x}\right)+y^{g r}\left(\mu, \alpha_{y}\right)= \\
& =z^{g r}\left(\mu, \alpha_{x}, \alpha_{y}\right), \quad \mu, \alpha_{x}, \alpha_{y} \in[0,1]
\end{aligned}
$$

For example, if $X$ is trapezoidal $\mathrm{MF}(1,3,4,5)$ :

$$
x^{g r}=(1+2 \mu)+(4-3 \mu) \alpha_{x},
$$

and $Y$ is trapezoidal MF $(1,2,3,4)$ :

$$
y^{g r}=(1+\mu)+(3-2 \mu) \alpha_{y}
$$

then $z^{g r}$ is given by:

$$
\begin{gathered}
z^{g r}=(2+3 \mu)+(4-3 \mu) \alpha_{x}+(3-2 \mu) \alpha_{y}, \\
\mu, \alpha_{x}, \alpha_{y} \in[0,1] .
\end{gathered}
$$

The 4D-solution (16) exists in the space which cannot be seen. Therefore we can be interested in its low dimensional representations. Frequently, the 2D-representation in the form of span $s\left(z^{g r}\right)$ is determined. It can be found with known methods of function examination:

$$
s\left(z^{g r}\right)=\left[\min _{\alpha_{x}, \alpha_{y}} z^{g r}\left(\mu, \alpha_{x}, \alpha_{y}\right), \max _{\alpha_{x}, \alpha_{y}} z^{g r}\left(\mu, \alpha_{x}, \alpha_{y}\right)\right] .
$$

In the case discussed, extrema of (16) do not lie inside, but on boundaries of the result domain. The minimum corresponds to $\alpha_{x}=\alpha_{y}=0$ and the maximum to $\alpha_{x}=\alpha_{y}=1$. The span of the 4D-result granule (16) is given by:

$$
s\left(z^{g r}\right)=[2+3 \mu, 9-2 \mu], \quad \mu \in[0,1] .
$$

The span (17) is not the addition result. The addition result has the form of 4D-function (16). The span is only a 2D-information about the maximal uncertainty of the result.

\section{Subtraction of two independent fuzzy intervals}

$$
\begin{aligned}
X-Y & =Z: x^{g r}\left(\mu, \alpha_{x}\right)-y^{g r}\left(\mu, \alpha_{y}\right)= \\
& =z^{g r}\left(\mu, \alpha_{x}, \alpha_{y}\right), \quad \mu, \alpha_{x}, \alpha_{y} \in[0,1]
\end{aligned}
$$

For example, if $X$ and $Y$ are trapezoidal MF (14) and (15) then the result is given by:

$$
\begin{gathered}
z^{g r}=\mu+(4-3 \mu) \alpha_{x}-(3-2 \mu) \alpha_{y}, \\
\mu, \alpha_{x}, \alpha_{y} \in[0,1] .
\end{gathered}
$$

If we are interested in the span representation $s\left(z^{g r}\right)$ of the $4 \mathrm{D}$-subtraction result, then it can be determined from:

$$
\begin{gathered}
s\left(z^{g r}\right)=\left[\min _{\alpha_{x}, \alpha_{y}} z^{g r}, \max _{\alpha_{x}, \alpha_{y}} z^{g r}\right]=[-3+3 \mu, 4-2 \mu], \\
\mu \in[0,1] .
\end{gathered}
$$

The span (20) of $z^{g r}$ (19) corresponds to $\alpha_{x}=0, \alpha_{y}=1$ for $\min z^{g r}$ and $\alpha_{x}=1, \alpha_{y}=0$ for $\max z^{g r}$.

\section{Multiplication of two independent fuzzy intervals}

$$
\begin{gathered}
X \cdot Y=Z: x^{g r}\left(\mu, \alpha_{x}\right) \cdot y^{g r}\left(\mu, \alpha_{y}\right)=z^{g r}\left(\mu, \alpha_{x}, \alpha_{y}\right), \\
\mu, \alpha_{x}, \alpha_{y} \in[0,1]
\end{gathered}
$$

For example, if $X$ and $Y$ are trapezoidal MF (14) and (15) then the multiplication result $z^{g r}$ is expressed by:

$$
\begin{aligned}
z^{g r}= & x^{g r} \cdot y^{g r}=\left[(1+2 \mu)+(4-3 \mu) \alpha_{x}\right] . \\
& \cdot\left[(1+\mu)+(3-2 \mu) \alpha_{y}\right], \quad \mu, \alpha_{x}, \alpha_{y} \in[0,1]
\end{aligned}
$$

Formula (22) describes the full 4D-result of the multiplication. If we are interested in the 2D simplified representation of this result in the form of a span $s\left(z^{g r}\right)$ then formula (23) should be used.

$$
\begin{aligned}
s\left(z^{g r}\right)= & {\left[\min _{\alpha_{x}, \alpha_{y}} z^{g r}, \max _{\alpha_{x}, \alpha_{y}} z^{g r}\right]=[(1+2 \mu)(1+\mu),} \\
& (5-\mu)(4-\mu)], \quad \mu \in[0,1] .
\end{aligned}
$$

Figure 4 shows the MF of the span representation of the multiplication result.

\section{Division $X / Y$ of two independent fuzzy intervals, $0 \notin Y$}

$$
\begin{gathered}
X / Y=Z: x^{g r}\left(\mu, \alpha_{x}\right) / y^{g r}\left(\mu, \alpha_{y}\right)=z^{g r}\left(\mu, \alpha_{x}, \alpha_{y}\right), \\
\mu, \alpha_{x}, \alpha_{y} \in[0,1]
\end{gathered}
$$

For example, if $X$ and $Y$ are trapezoidal MF (14) and (15) then the division result $z^{g r}$ is given by:

$$
\begin{gathered}
z^{g r}=x^{g r} / y^{g r}=\frac{(1+2 \mu)+(4-3 \mu) \alpha_{x}}{(1+\mu)+(3-2 \mu) \alpha_{y}}, \\
\mu, \alpha_{x}, \alpha_{y} \in[0,1] .
\end{gathered}
$$




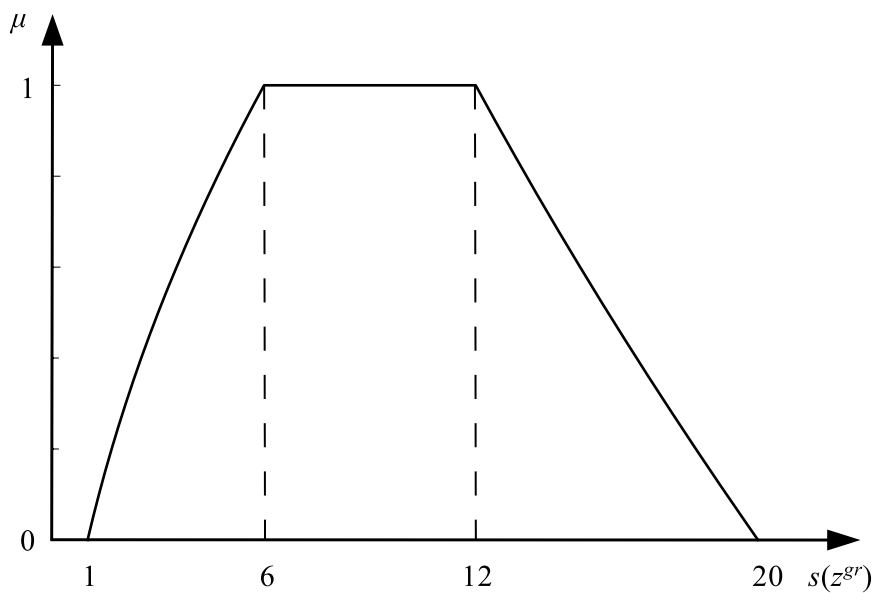

Fig. 4. MF of the span representation of the 4D multiplication result (22)

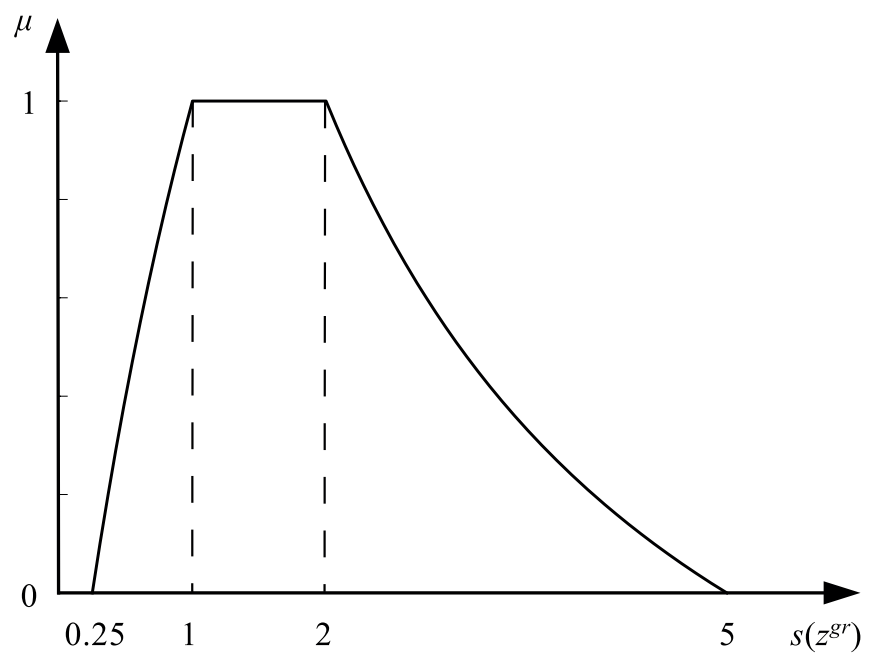

Fig. 5. Span representation of the 4D division result (25)

The span representation $s\left(z^{g r}\right)$ of the result (25) is expressed by (26) and is shown in Fig. 5.

$$
\begin{aligned}
s\left(z^{g r}\right) & =\left[\min _{\alpha_{x}, \alpha_{y}} z^{g r}, \max _{\alpha_{x}, \alpha_{y}} z^{g r}\right] \\
& =\left[\frac{1+2 \mu}{4-\mu}, \frac{5-\mu}{1+\mu}\right], \quad \mu \in[0,1] .
\end{aligned}
$$

The solution granule of the division (25) is 4-dimensional, so it cannot be presented in its full space. However, it can be shown in a simplified way, in the $X \times Y \times Z 3 \mathrm{D}$-space, without $\mu$-coordinate. Figure 6 presents surfaces for constant $\mu=0$ and $\mu=1$ values.

As Fig. 6 shows, the solution granule (25) is uniform. This results from the fact that the divisor does not contain zero. As it will be shown further on, division results can be discontinuous and multi-granular in more complicated cases.

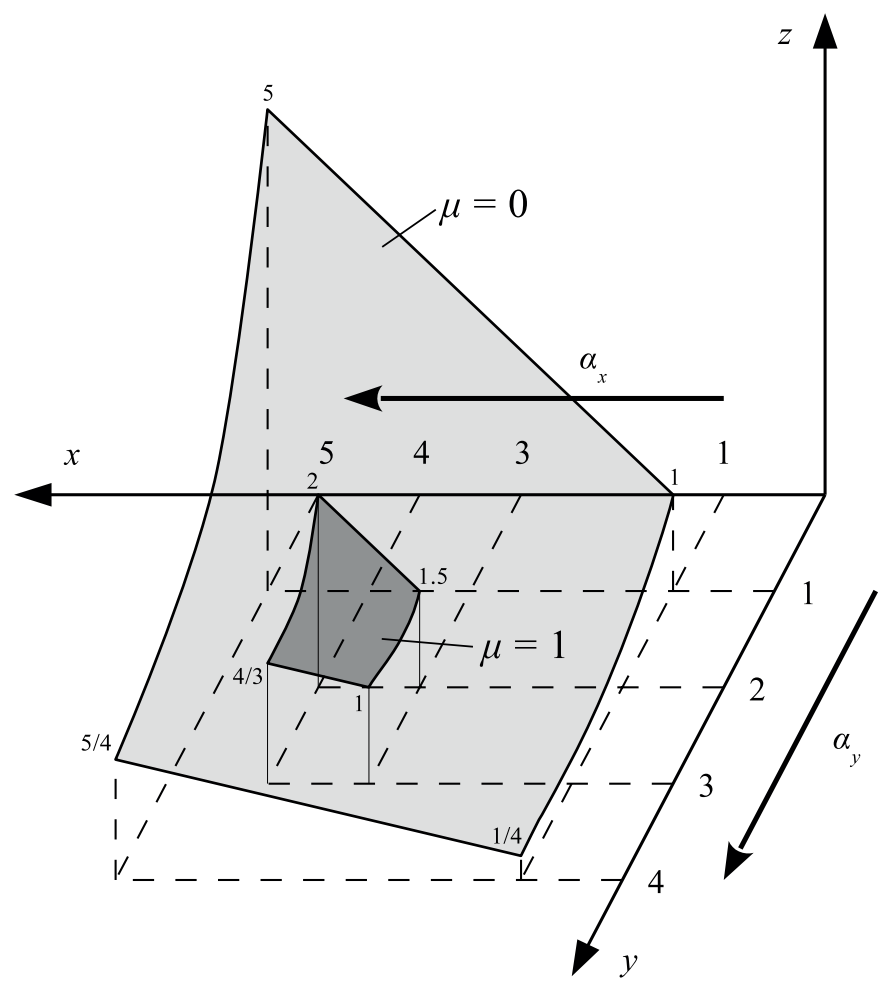

Fig. 6. Simplified view of the 4D-solution granule $(25) z^{g r}(x, y, z)$ in 3D-space $X \times Y \times Z$, without $\mu$-coordinate

\section{Mathematical properties of MD RDM fuzzy arithmetic}

\section{Commutativity}

For any fuzzy intervals $X$ and $Y$, equations (27) and (28) are true.

$$
\begin{aligned}
X+Y & =Y+X \\
X Y & =Y X
\end{aligned}
$$

\section{Associativity}

For any fuzzy intervals $X, Y$ and $Z$, equations (29) and (30) are true.

$$
\begin{aligned}
X+(Y+Z) & =(X+Y)+Z \\
X(Y Z) & =(X Y) Z
\end{aligned}
$$

\section{Neutral element of addition and multiplication}

In MD RDM FA, there exist additive and multiplicative neutral elements such as the degenerate interval 0 and 1 for any interval $X$.

$$
\begin{gathered}
X+0=0+X=X \\
X \cdot 1=1 \cdot X=X
\end{gathered}
$$




\section{Inverse elements}

In MD RDM FA, fuzzy interval:

$$
\begin{aligned}
-X:-x^{g r}= & -[a+(b-a) \mu]-[(d-a) \\
& -\mu(d-a+b-c)] \alpha_{x}, \quad \alpha_{x} \in[0,1],
\end{aligned}
$$

is an additive inverse element of fuzzy interval:

$$
\begin{aligned}
X: x^{g r} & =[a+(b-a) \mu]+[(d-a)- \\
& -\mu(d-a+b-c)] \alpha_{x}, \quad \alpha_{x} \in[0,1] .
\end{aligned}
$$

If parameters of two fuzzy intervals $X$ and $Y$ are equal: $a_{x}=a_{y}, b_{x}=b_{y}, c_{x}=c_{y}, d_{x}=d_{y}$, then the interval $-Y$ is the additive inverse interval of $X$, when also inner RDM-variables are equal: $\alpha_{x}=\alpha_{y}$. It means full coupling (correlation) of both uncertain values $x$ and $y$ modelled by intervals.

Assuming that $0 \notin X$, a multiplicative inverse element of the fuzzy interval $X$ is equal in MD RDM FA:

$$
\begin{aligned}
& \frac{1}{X}: \frac{1}{x^{g r}}=\frac{1}{[a+(b-a) \mu]+[(d-a)-\mu(d-a+b-c)] \alpha_{x}}, \\
& \alpha_{x} \in[0,1] .
\end{aligned}
$$

If parameters of two fuzzy intervals $X$ and $Y$ are equal: $a_{x}=a_{y}, b_{x}=b_{y}, c_{x}=c_{y}, d_{x}=d_{y}$, then the interval $1 / Y$ is the multiplicative inverse interval of $X$ only when also inner RDM-variables are equal: $\alpha_{x}=\alpha_{y}$. It means full coupling (correlation) of both uncertain values $x$ and $y$ modelled by intervals. Such full or partial correlation of uncertain variables occurs in many real problems.

\section{Sub-distributivity law}

The sub-distributivity law holds in MD RDM FA:

$$
X(Y+Z)=X Y+X Z \text {. }
$$

The consequence of this law is the possibility of formula transformations. They do not change the calculation result.

\section{Cancellation law for addition and multiplication}

Cancellation laws (34) and (35) hold in MD RDM FA:

$$
\begin{aligned}
X+Z & =Y+Z \Rightarrow X=Y, \\
X Z & =Y Z \Rightarrow X=Y .
\end{aligned}
$$

\section{Multi-granularity phenomenon in division of fuzzy intervals}

As it was presented in Section 2, result in the form of only one granule is achieved during dividing fuzzy intervals $X$ and $Y$, $0 \notin Y$. However, it will be shown further on that the solution is multi-granular if a denominator set contains 0 .

Three examples below describe problems in which division by fuzzy denominator containing 0 occurs and in which solutions will be multi-granular.
Example 1. A company produces a food product. The production cost $c_{1}$ per $1 \mathrm{~kg}$ of the product is uncertain because it depends on current prices of components changing from month to month and on negotiations with many suppliers. Price $p_{1}$ of $1 \mathrm{~kg}$ of the product is also uncertain because it is also a result of negotiations with customers (supermarkets, shops, dealers). It also depends on volume of purchased product. Profit $\mathrm{Pr}$ of the company is equal to the difference between incomes and costs: $\operatorname{Pr}=I n-C s$. The global income can be calculated as: In $=n \cdot p_{1}$, where $n$ is the number of kilograms of the product. General costs of production are equal to $C s=100000+n \cdot c_{1}$, where the number 100000 describes fixed costs of the company.

Hence, the company profit $\operatorname{Pr}$ is expressed by equation:

$$
\begin{aligned}
\operatorname{Pr} & =n \cdot p_{1}-\left(100000+n \cdot c_{1}\right)= \\
& =n \cdot\left(p_{1}-c_{1}\right)-100000 .
\end{aligned}
$$

In the next year, the company managers plan to achieve general profit 'about 5500000 ' defined by the triangular fuzzy number (5 $300000,5500000,5700000)$. Company experts evaluated the $\operatorname{cost} c_{1}$ as 'about 1 ' defined by the triangular fuzzy number $(0.9,1,1.1)$ and the price $p_{1}$ as 'about 1.2 ' expressed by $(1,1.2$, 1.4). How many kilograms $n$ of the product should the company produce in the next year?

To obtain the answer, the following calculation should be realized:

$$
\begin{aligned}
n & =\frac{\operatorname{Pr}-100000}{p_{1}-c_{1}}= \\
& =\frac{\left(5.3 \cdot 10^{6}, 5.5 \cdot 10^{6}, 5.7 \cdot 10^{6}\right)+10^{5}}{(1,1.2,1.4)-(0.9,1.0,1.1)}
\end{aligned}
$$

Denominator of the formula (36) contains 0 in a hidden way. Example 1 corresponds to Case 3 of division described in the paper further on.

Example 2. An oil rafinery produces regular and super gasoline using two production lines. Line 1 produces gasoline according to an older process and line 2 according to a newer process. The older process produces about 6.0 units of regular and about 5.5 units of super gasoline in one run. The newer process produces about 8.5 units of regular and about 8 units of super gasoline. Production results are uncertain because 3 types of crude oils used in the production (supplied by few companies) are of different quality.

Uncertain results can be described by the following triangle fuzzy numbers:

$$
\begin{aligned}
& \text { about } 5.5=\tilde{5.5}=(5.2,5.5,5.8), \\
& \text { about } 6.0=\tilde{6.0}=(5.7,6.0,6.3), \\
& \text { about } 8.0=\tilde{8.0}=(7.8,8.0,8.2), \\
& \text { about } 8.5=\tilde{8.5}=(8.2,8.5,8.8) .
\end{aligned}
$$

In the next month the company predicts to get contracts for production of about 500 units of regular and about 350 units of 
super gasoline, where uncertain values are described by triangle fuzzy numbers:

$$
\begin{aligned}
& \text { about } 500=5 \tilde{0} 0=(480,500,520), \\
& \text { about } 350=3 \tilde{5} 0=(330,350,370) .
\end{aligned}
$$

Let us denote by $x_{1}$ - the number of production runs of line 1 (older process) and by $x_{2}$ - the number of runs of line 2 (newer process). After $x_{1}$ runs of line 1 and $x_{2}$ runs of line 2 amount of regular gasoline will be $\tilde{6} x_{1}+8.5 x_{2}$ and of super gasoline $5.5 x_{1}+\tilde{8} x_{2}$. How many runs $x_{1}$ of line 1 and $x_{2}$ of line 2 are necessary to fulfil the contract commitments?

The results $x_{1}$ and $x_{2}$ have not to be integers because it is possible to organize fractional runs that use raw materials in an appropriate proportion. To determine results $x_{1}$ and $x_{2}$, following equation system has to be solved:

$$
\left[\begin{array}{cc}
\tilde{6} & \tilde{8.5} \\
5.5 & \tilde{8}
\end{array}\right]\left[\begin{array}{l}
x_{1} \\
x_{2}
\end{array}\right]=\left[\begin{array}{c}
5 \tilde{0} 0 \\
3 \tilde{5} 0
\end{array}\right] \text {. }
$$

The results are given by $x_{1}=D_{1} / D$ and $x_{2}=D_{2} / D$ where: $D=\tilde{6} \cdot \tilde{8}-\tilde{5.5} \cdot \tilde{8.5}, D_{1}=5 \tilde{0} 0 \cdot \tilde{8}-3 \tilde{5} 0 \cdot \tilde{8.5}$ and $D_{2}=\tilde{6} \cdot 3 \tilde{5} 0-$ $-5.5 \cdot 5 \tilde{0} 0$.

Determinant $D=(5.7,6,6.3) \cdot(7.8,8,8.2)-(5.2,5.5,5.8) \cdot$ $\cdot(8.2,8.5,8.8)$ contains 0 as one of possible values. Therefore the solution of the problem corresponds to Case 5 presented further on in the paper and it will be multi-granular.

Example 3. The ferry departs from point A on the southern bank of the river and has to get to any place of the concrete quay on the northern bank between points $\mathrm{B}_{1}$ and $\mathrm{B}_{2}$, the distance $l_{\mathrm{B} 1 \mathrm{~B} 2}=1 \mathrm{~km}$. The remaining northern bank of the river is not suitable for mooring, Fig. 7. An average speed of the river depends on the water level and varies in the range $V_{1} \in[2,4] \mathrm{km} / \mathrm{h}$. The ferry has to reach the opposite bank within time $T=1$ hour. The distance to the bank is $d=1 \mathrm{~km}$. We must specify an angle $\beta$ and a speed $V_{2}$ in relation to water, assuming that the ferry should reach the point $\mathrm{B}$ located between points $\mathrm{B}_{1}$ and $\mathrm{B}_{2}$.

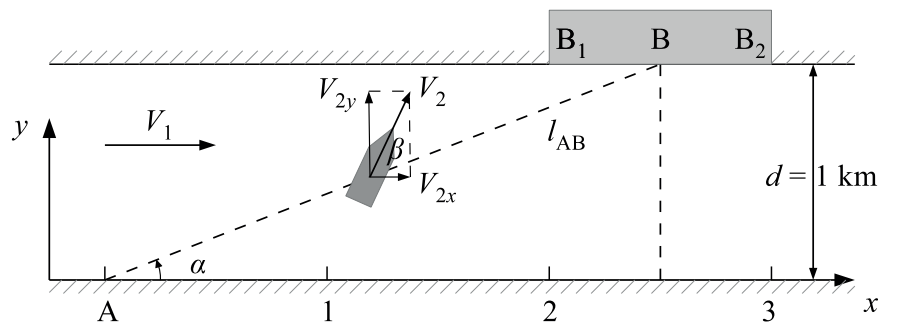

Fig. 7. An illustration to Example 3

A vertical component of the relative speed $V_{2}$ should satisfy the condition:

$$
V_{2 y}=\frac{d}{T}=\frac{1}{1}=1
$$

As the ferry (apart from its own move) is floated by the water with a speed $V_{1}$, a horizontal component of the relative speed $V_{2}$ should satisfy the next condition:

$$
\left(V_{2 x}+V_{1}\right) T=l_{A B} \cdot \cos \alpha
$$

Thus, the total speed $V_{2}$ of the ferry can be calculated as:

$$
V_{2}=\sqrt{V_{2 x}^{2}+V_{2 y}^{2}}=\sqrt{\left(\frac{l_{A B} \cdot \cos \alpha}{T}-V_{1}\right)^{2}}+\left(\frac{d}{T}\right)^{2},
$$

and the course angle of the ferry is defined as:

$$
\operatorname{tg} \beta=\frac{V_{2 y}}{V_{2 x}}=\frac{l_{A B} \cdot \sin \alpha}{l_{A B} \cdot \cos \alpha-V_{1} T} .
$$

The value of $l_{A B} \cdot \sin \alpha$ always equals 1 and the value of $l_{A B} \cdot \cos \alpha$ must be included in the interval [2, 3], Fig. 7. Taking into account all known values, we obtain new interval dependencies from equations (40) and (41):

$$
\begin{gathered}
V_{2}=\sqrt{\left(\frac{[2,3]}{1}-[2,4]\right)^{2}+\left(\frac{1}{1}\right)^{2}}= \\
=\sqrt{([2,3]-[2,4])^{2}+1}, \\
\operatorname{tg} \beta=\frac{1}{[2,3]-[2,4]} .
\end{gathered}
$$

Formulas (42) and (43) determine the relative speed $V_{2}$ and the course angle $\beta$ of the ferry and it can be seen that they are coupled. The RDM arithmetic, in contrast to the standard interval arithmetic, allows for taking into account such couplings. The interval $[2,3]$, describing the concrete quay on the northern bank between points $\mathrm{B}_{1}$ and $\mathrm{B}_{2}$, is defined as:

$$
l_{A B} \cdot \cos \alpha=2+\alpha_{B 1 B 2}, \quad \alpha_{B 1 B 2} \in[0,1]
$$

and the interval describing values of the river speed is:

$$
V_{1}=2+2 \alpha_{V 1}, \quad \alpha_{V 1} \in[0,1] .
$$

Finally, possible values of $V_{2}$ and $\operatorname{tg} \beta$ are described by equations:

$$
\begin{gathered}
V_{2}=\sqrt{\left(2+\alpha_{B 1 B 2}\right)+\left(2+2 \alpha_{V 1}\right)^{2}+1}, \\
\alpha_{B 1 B 2}, \alpha_{V 1} \in[0,1]
\end{gathered}
$$

$$
\operatorname{tg} \beta=\frac{1}{\left(2+\alpha_{B 1 B 2}\right)-\left(2+2 \alpha_{V 1}\right)}=\frac{1}{\alpha_{B 1 B 2}-2 \alpha_{V 1}} .
$$

Formulas (46) and (47) also show that the speed $V_{2}$ and the angle $\beta$ are coupled. The speed should be calculated according to the chosen course angle. 


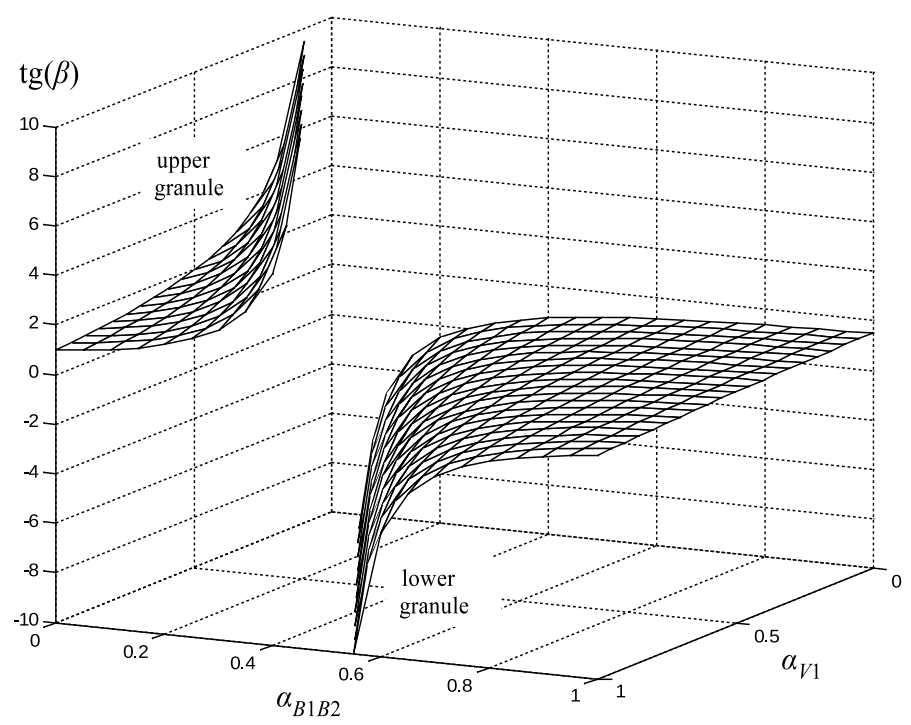

Fig. 8. Two granules representing subsets of possible solutions; the upper granule corresponds to the acute course angle of the ferry $\left(\beta<90^{\circ}\right)$ and the lower corresponds to the obtuse angle $\left(\beta>90^{\circ}\right)$

From the point of view of this paper, formula (47) is the most interesting. Figure 8 presents two separate granules of its possible solutions. A positive value of $\operatorname{tg} \beta$ means the movement of the ferry in the direction of the river current, while a negative value means movement in the opposite direction.

4.1. Case 1. Let us assume that two triangular fuzzy numbers $X_{A}=(1,2,3)$ and $X_{B}=(-3,0,1)$ have to be divided (Fig. 9). How to calculate the quotient $Z=X_{A} / X_{B}$ ?

Is it possible to divide (about 2)/(about 0)? It is possible, but after excluding the value $x_{B}=0$. For this purpose, FN (about $0)$ has to be decomposed into two components: $X_{B}=X_{B^{-}} \cup X_{B^{+}}$, where $X_{B^{-}}$is the triangle fuzzy number $\left(-3,0^{-}, 0^{-}\right)$and $X_{B^{+}}$is the triangle fuzzy number $\left(0^{+}, 0^{+}, 1\right)$ (Fig. 10).
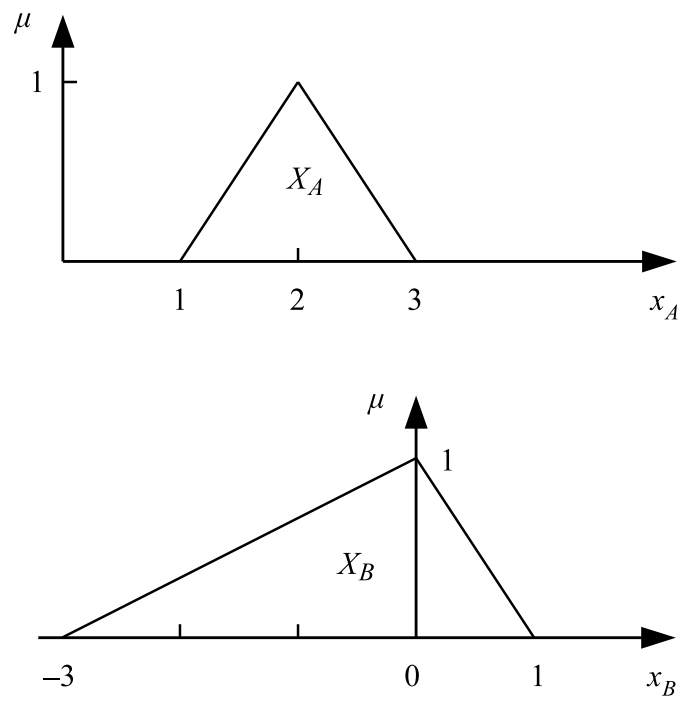

Fig. 9. Fuzzy numbers $X_{A}$ (about 2) and $X_{B}$ (about 0)

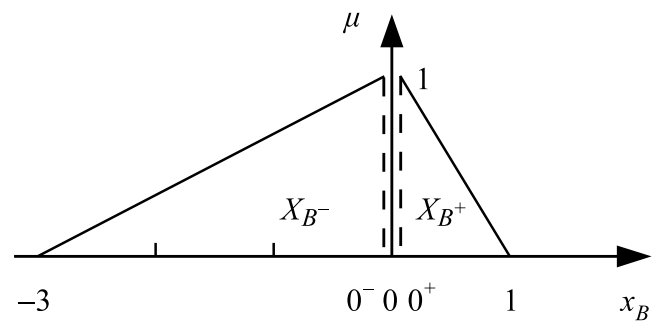

Fig. 10. Decomposition of the fuzzy number $X_{B}$ into two components $X_{B^{-}}$and $X_{B^{+}}$

The quotient $X_{A} / X_{B}$ can be presented in the form:

$$
X_{A} / X_{B}=\left(X_{A} / X_{B^{-}}\right) \cup\left(X_{A} / X_{B^{+}}\right) .
$$

From equation (10) and Fig. 9, horizontal MFs of sets $X_{A}$, $X_{B^{-}}, X_{B^{+}}$can be found:

$$
\begin{aligned}
X_{A}: & x_{A}=(1+\mu)+2(1-\mu) \alpha_{X A}, \\
X_{B^{-}}: & x_{B^{-}}=-3+\left(0^{-}+3\right) \mu+\left(0^{-}+3\right)(1-\mu) \alpha_{X B^{-}}, \\
X_{B^{+}}: & x_{B^{+}}=0^{+}+\left(1-0^{+}\right)(1-\mu) \alpha_{X B^{+}}, \\
& \mu, \alpha_{X A}, \alpha_{X B^{-}}, \alpha_{X B^{+}} \in[0,1] .
\end{aligned}
$$

Taking into account (49), we get the formula for the division:

$$
\begin{aligned}
& X_{A} / X_{B}: x_{A} / x_{B}=x_{A} / x_{B^{-}} \cup x_{A} / x_{B^{+}}= \\
& =\frac{(1+\mu)+2(1-\mu) \alpha_{X A}}{-3+\left(0^{-}+3\right) \mu+\left(0^{-}+3\right)(1-\mu) \alpha_{X B^{-}}} \cup \\
& \cup \frac{(1+\mu)+2(1-\mu) \alpha_{X A}}{0^{+}+\left(1-0^{+}\right)(1-\mu) \alpha_{X B^{+}}}, \\
& \mu, \alpha_{X A}, \alpha_{X B^{-}}, \alpha_{X B^{+}} \in[0,1] .
\end{aligned}
$$

According to (50), the true value of the quotient $x_{A} / x_{B}$ is located in the granule $x_{A} / x_{B^{-}}$or $x_{A} / x_{B^{+}}$. Both quotients are monotonic, so their extrema can be found on boundaries of possible solutions sets. Table 1 and 2 present values of quotients in corners of solution sets for $\mu=0$.

Solution granules $x_{A} / x_{B^{-}}$and $x_{A} / x_{B^{+}}$are functions existing in 4D-space because $x_{A} / x_{B^{-}}=f\left(\mu, \alpha_{x A}, \alpha_{x B^{-}}\right)$and $x_{A} / x_{B^{+}}=$ $=f_{2}\left(\mu, \alpha_{x A}, \alpha_{x B^{+}}\right)$. Hence, they cannot be fully visualized. However, the granules can be visualized partly in the 3D-space which corresponds to various levels of $\mu$-cuts, e.g. $\mu=0$,

Table 1

Values of $x_{A} / x_{B^{-}}$in corners of the solution space, level $\mu=0$

\begin{tabular}{l|rrrc}
\hline$\alpha_{X A}$ & 0 & 0 & 1 & 1 \\
$\alpha_{X B^{-}}$ & 0 & 1 & 0 & 1 \\
\hline$x_{A}$ & 1 & 1 & 3 & 3 \\
$x_{B^{-}}$ & -3 & $0^{-}$ & -3 & $0^{-}$ \\
\hline$x_{A} / x_{B^{-}}$ & $-1 / 3$ & $-\infty$ & -1 & $-\infty$ \\
\hline
\end{tabular}


Table 2

Values of $x_{A} / x_{B^{+}}$in corners of the solution space, level $\mu=0$

\begin{tabular}{l|cccc}
\hline$\alpha_{X A}$ & 0 & 0 & 1 & 1 \\
$\alpha_{X B^{+}}$ & 0 & 1 & 0 & 1 \\
\hline$x_{A}$ & 1 & 1 & 3 & 3 \\
$x_{B^{+}}$ & $0^{+}$ & 1 & $0^{+}$ & 1 \\
\hline$x_{A} / x_{B^{+}}$ & $+\infty$ & 1 & $+\infty$ & 3 \\
\hline
\end{tabular}

$\mu=0.5$, etc. Figure 11 shows 2 granules of the quotient $x_{A} / x_{B}$ in the 3D-space.

Granules of the quotient $z=x_{A} / x_{B}$ can be presented also in a more simplified way, in the 2D-space: $Z \times X_{A}$ (Fig. 12).

As Fig. 12 shows, the result of the division of two fuzzy numbers: $X_{A}$ determined by triple $(1,2,3)$ and $X_{B}$ that contains zero $(-3,0,1)$ is not one, single and compact granule. The result consists of two distinctly separated granules. The smallest separation occurs for the cut-level $\mu=0$. With increasing $\mu$-value, the separation of granules $Z_{-}$and $Z_{+}$increases. The separation approaches infinity for $\mu=1$.

In the considered example of the division $X_{A} / X_{B}$, zero occurs explicitly in the denominator of the number $X_{B}$. However, in many calculation tasks, zero does not occur in denominators openly but in a more or less secret way. Then we cannot be aware of its occurrence. It will be shown in next examples.

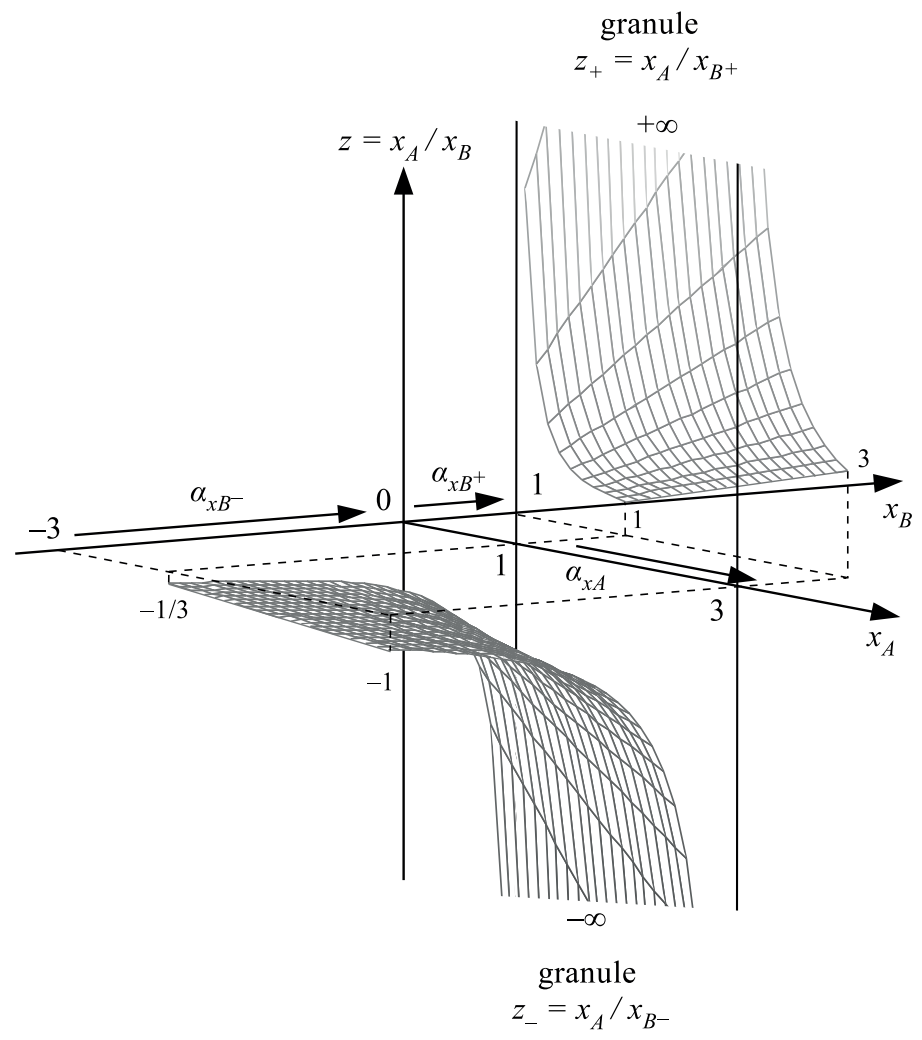

Fig. 11. Visualization of two granules of the quotient $a=x_{A} / x_{B}$, formula (50), in 3D-space for the cut $\mu=0$

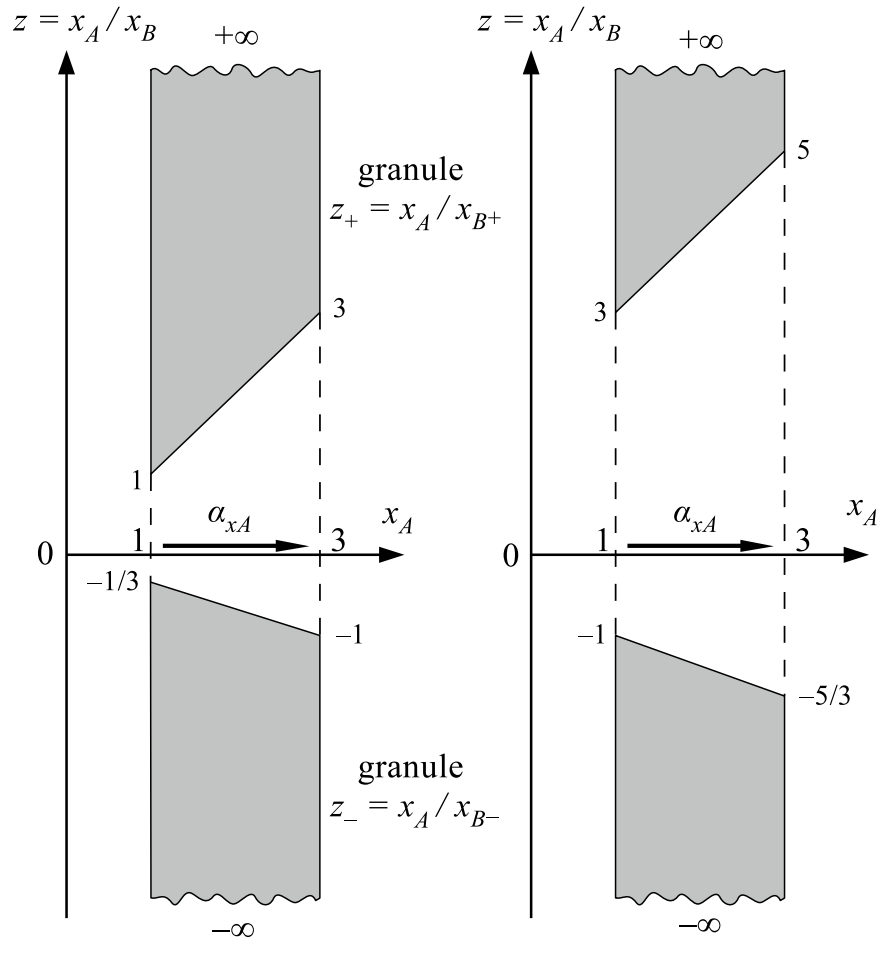

cut level $\mu=0$

cut level $\mu=0.5$

Fig. 12. Granules of the quotient $z=x_{A} / x_{B}$, formula (50), in the 2D-space for cut-level $\mu=0$ and $\mu=0.5$

4.2. Case 2. Let us consider the case of a division in which 3 fuzzy intervals $A, B, C$ take part:

$$
\frac{A}{B-C}=\frac{[1,2,3]}{[2,3,4]-[3,4,5]}=Z
$$

None of intervals contains zero. However, though not directly, the denominator $B-C$ does. Horizontal MFs of $A, B$ and $C$ will be formulated (52) to calculate the division result.

$$
\begin{gathered}
A: x_{A}=(1+\mu)+2(1-\mu) \alpha_{X A}, \\
B: x_{B}=(2+\mu)+2(1-\mu) \alpha_{X B}, \\
C: x_{C}=(3+\mu)+2(1-\mu) \alpha_{X C}, \\
\mu, \alpha_{X A}, \alpha_{X B}, \alpha_{X C} \in[0,1]
\end{gathered}
$$

After using horizontal MFs in (51) we obtain

$$
\begin{gathered}
\frac{A}{B-C}=Z: \frac{x_{A}^{g r}}{x_{B}^{g r}-x_{C}^{g r}}=z^{g r}, \\
z^{g r}=\frac{(1+\mu)+2(1-\mu) \alpha_{X A}}{-1+2(1-\mu)\left(\alpha_{X B}-\alpha_{X C}\right)}, \\
\mu, \alpha_{X A}, \alpha_{X B}, \alpha_{X C} \in[0,1] .
\end{gathered}
$$


For the cut-level $\mu=0$ formula (53) takes form:

$$
\begin{aligned}
& Z: z^{g r}=\frac{1+2 \alpha_{X A}}{-1+2\left(\alpha_{X B}-\alpha_{X C}\right)}, \\
& \alpha_{X A}, \alpha_{X B}, \alpha_{X C} \in[0,1], \quad \alpha_{X B}-\alpha_{X C} \neq 0.5 .
\end{aligned}
$$

As can be seen from (54), the division result $z^{g r}=f\left(\alpha_{X A}\right.$, $\left.\alpha_{X B}, \alpha_{X C}\right)$ exists in the 4D-space and cannot be fully visualized. However, in a simplified way, it can be shown in 2D-space (Fig. 13).

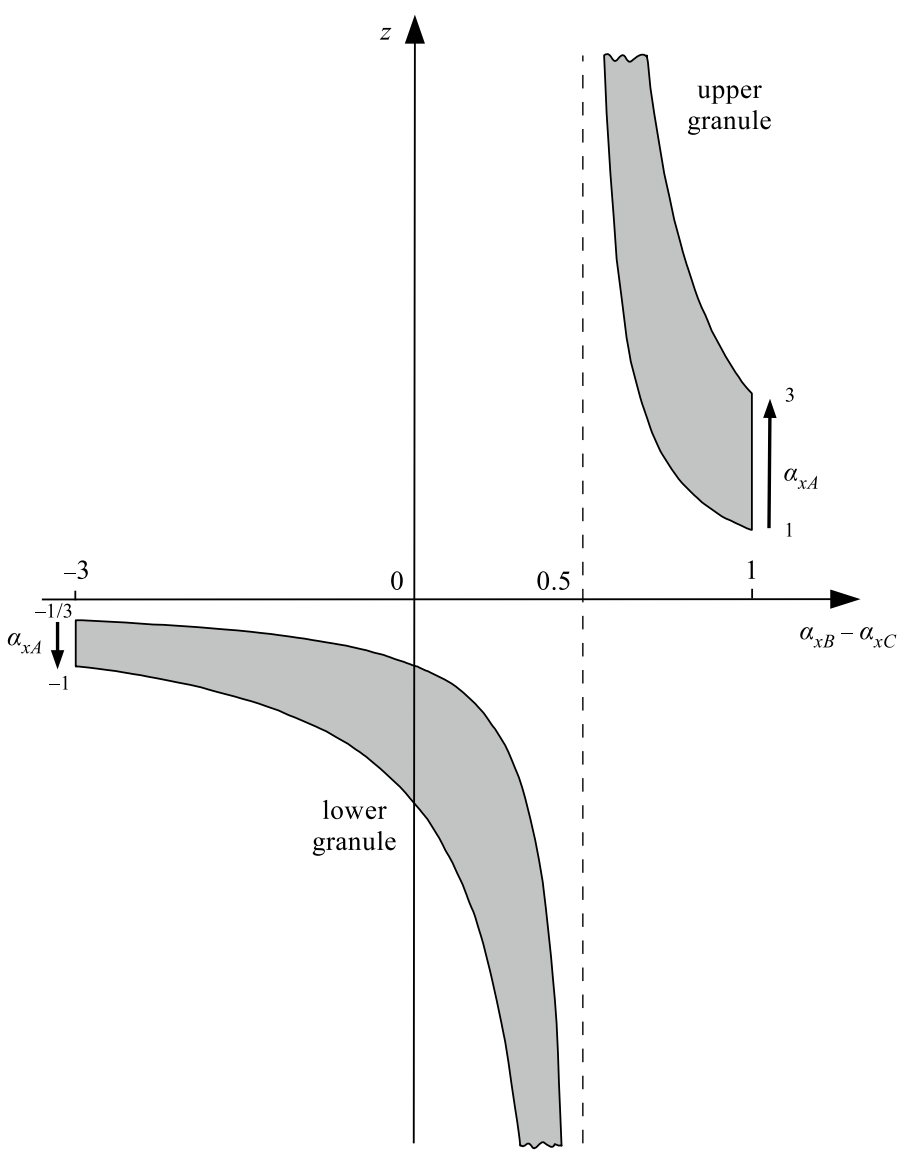

Fig. 13. Simplified 2D-visualization of two separate solution granules of division $A /(B-C)$ on the cut-level $\mu=0$, formula (54); values $z \in[-1 / 3,1]$ are impossible

As Fig. 13 shows, solution values $z \in[-1 / 3,1]$ are impossible for the cut-level $\mu=0$. It can be easily checked by use of formula (54). If the value $z=0$ is possible, equation (55) should also have a solution.

$$
\frac{1+2 \alpha_{X A}}{-1+2\left(\alpha_{X B}-\alpha_{X C}\right)}=0, \quad \alpha_{X B}-\alpha_{X C} \neq 0.5
$$

However, since $\alpha_{X A} \in[0,1]$, the division result $z=0$ is impossible. A similar situation concerns other $\mu$-levels.
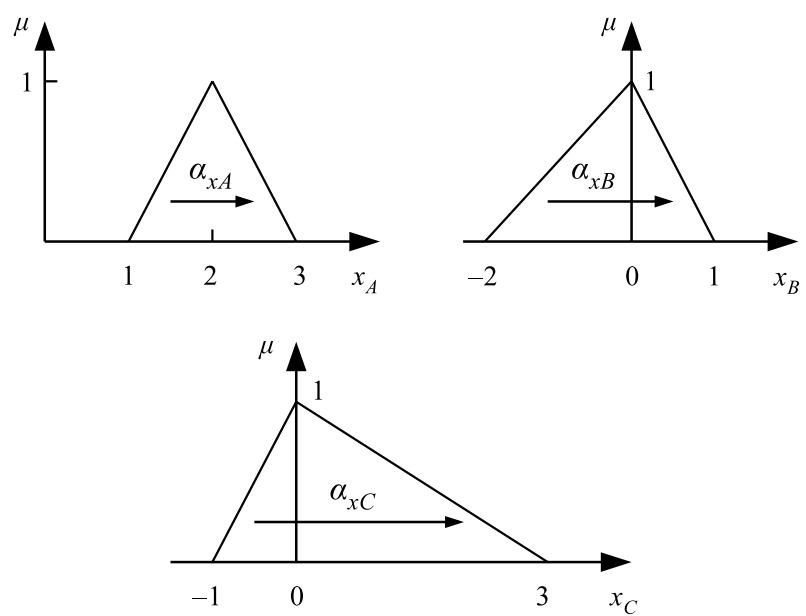

Fig. 14. Fuzzy intervals $A, B, C$ taking part in the division (56)

4.3. Case 3. Let us consider a more complicated division case, where two fuzzy intervals containing zero appear in the denominator. Fuzzy intervals are shown in Fig. 14.

$$
\frac{A}{B C}=\frac{[1,2,3]}{[-2,0,1][-1,0,3]}, \quad x_{B} \neq 0, x_{C} \neq 0
$$

Since zero-values of uncertain variables $x_{B}$ and $x_{C}$ cannot take part in the division operation, these values have to be removed from the intervals (Fig. 15).
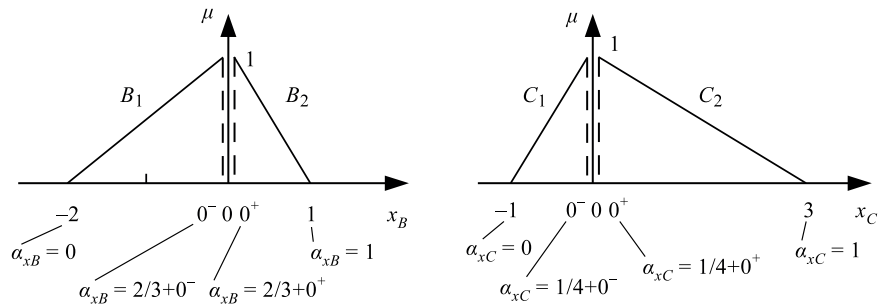

Fig. 15. Removing zero-values and partition of fuzzy intervals $B$ and $C$ occurring in the denominator of the division (56)

$0^{-}$is a very small, negative value approaching 0 . Similarly $0^{+}$is a very small positive value approaching 0 . Symbolically, the division operation can be decomposed into 4 components:

$$
\begin{aligned}
\frac{A}{B C}=Z & =\frac{A}{B_{1} C_{1}} \cup \frac{A}{B_{1} C_{2}} \cup \frac{A}{B_{2} C_{1}} \cup \frac{A}{B_{2} C_{2}}= \\
& =Z_{1} \cup Z_{2} \cup Z_{3} \cup Z_{4} .
\end{aligned}
$$

Particular component functions presented in Fig. 14 are described with the following horizontal MFs:

$$
\begin{aligned}
& A: x_{A}=(1+\mu)+2(1-\mu) \alpha_{X A}, \quad \mu, \alpha_{X A} \in[0,1], \\
& B: x_{B}=(1-\mu)\left(-2+3 \alpha_{X B}\right), \quad \mu, \alpha_{X B} \in[0,1], \\
& C: x_{C}=(1-\mu)\left(-1+4 \alpha_{X C}\right), \quad \mu, \alpha_{X C} \in[0,1] .
\end{aligned}
$$


Decomposed functions presented in Fig. 15 can be described by the following horizontal MFs:

$$
\begin{array}{ll}
B_{1}: x_{B}=(1-\mu)\left(-2+3 \alpha_{X B}\right), & \alpha_{X B} \in\left[0, \frac{2}{3}\right), \\
B_{2}: x_{B}=(1-\mu)\left(-2+3 \alpha_{X B}\right), & \alpha_{X B} \in\left(\frac{2}{3}, 1\right], \\
C_{1}: x_{C}=(1-\mu)\left(-1+4 \alpha_{X C}\right), & \alpha_{X C} \in\left[0, \frac{1}{4}\right), \\
C_{2}: x_{C}=(1-\mu)\left(-1+4 \alpha_{X C}\right), & \alpha_{X C} \in\left(\frac{1}{4}, 1\right] .
\end{array}
$$

Now, particular component division results $Z_{1}, Z_{2}, Z_{3}$ and $Z_{4}$ have to be found according to the general formula (56).

$$
\begin{aligned}
& Z_{1}=\frac{A}{B_{1} C_{1}}: \\
& z_{1}^{g r}=\frac{(1+\mu)+2(1-\mu) \alpha_{X A}}{\left[(1-\mu)\left(-2+3 \alpha_{X B}\right)\right]\left[(1-\mu)\left(-1+4 \alpha_{X C}\right)\right]} \\
& \alpha_{X A} \in[0,1], \alpha_{X B} \in\left[0, \frac{2}{3}\right), \alpha_{X C} \in\left[0, \frac{1}{4}\right)
\end{aligned}
$$

The division result $z_{1}^{g r}=f_{1}\left(\mu, \alpha_{X A}, \alpha_{X B}, \alpha_{X C}\right)$ is an information granule existing in a $5 \mathrm{D}$-space and hence it cannot be precisely visualized. However, it can be presented in a simplified

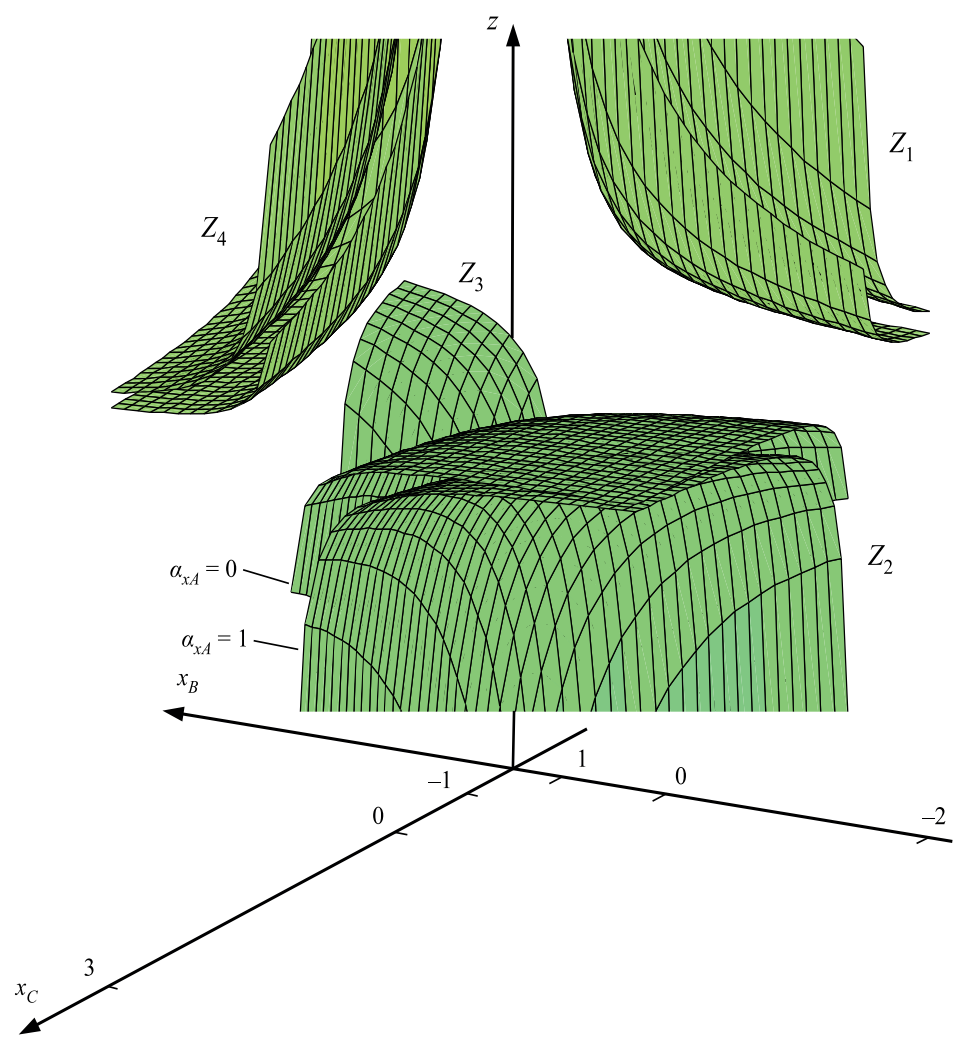

Fig. 16. Simplified visualization of $5 \mathrm{D}$ result granules $Z_{1}-Z_{4}$ of the division $Z=A /(B C)$ in a $3 \mathrm{D}$ subspace for the cut level $\mu=0$ way for particular levels of membership $\mu$. For the level $\mu=0$, formula (60) takes form:

$$
\begin{aligned}
& Z_{1}: z_{1}^{g r}=\frac{1+2 \alpha_{X A}}{\left[-2+3 \alpha_{X B}\right]\left[-1+4 \alpha_{X C}\right]}, \\
& \alpha_{X A} \in[0,1], \alpha_{X B} \in\left[0, \frac{2}{3}\right), \alpha_{X C} \in\left[0, \frac{1}{4}\right) .
\end{aligned}
$$

It should be noticed that in the considered domain of RDM variables $\alpha_{X A}, \alpha_{X B}, \alpha_{X C}$, the nominator and the denominator of formula (61) do not change their sign, i.e.: $1+2 \alpha_{X A}>0$, $-2+3 \alpha_{X B}<0,-1+4 \alpha_{X C}<0$. So, all possible division results contained in the granule $Z_{1}$ are positive. Analysis of formula (61) allows for simplified visualization of the granule of possible solutions $z_{1}^{g r}$, which is shown in Fig. 16.

Next, the component solution $Z_{2}$ of the division will be determined:

$$
\begin{aligned}
& Z_{2}=\frac{A}{B_{1} C_{2}}: \\
& z_{2}^{g r}=\frac{(1+\mu)+2(1-\mu) \alpha_{X A}}{\left[(1-\mu)\left(-2+3 \alpha_{X B}\right)\right]\left[(1-\mu)\left(-1+4 \alpha_{X C}\right)\right]}, \\
& \alpha_{X A} \in[0,1], \alpha_{X B} \in\left[0, \frac{2}{3}\right), \alpha_{X C} \in\left(\frac{1}{4}, 1\right] .
\end{aligned}
$$

Component $Z_{2}$ of the division is an information granule in the 5D-space. As before, for particular membership levels this granule can be visualized in a simplified way. For the level $\mu=0$, the granule is given by (63).

$$
\begin{aligned}
& Z_{2}: z_{2}^{g r}=\frac{1+2 \alpha_{X A}}{\left[-2+3 \alpha_{X B}\right]\left[-1+4 \alpha_{X C}\right]} \\
& \alpha_{X A} \in[0,1], \alpha_{X B} \in\left[0, \frac{2}{3}\right), \alpha_{X C} \in\left(\frac{1}{4}, 1\right]
\end{aligned}
$$

The granule $Z_{2}$ is visualized in Fig. 16. It can be noticed that granules $Z_{1}$ and $Z_{2}$ differ.

The component result $Z_{3}$ of the division is also an information granule in the $5 \mathrm{D}$-space. For various $\mu$-levels this result can be shown in the form:

$$
\begin{aligned}
& Z_{3}=\frac{A}{B_{2} C_{1}}: \\
& z_{3}^{g r}=\frac{(1+\mu)+2(1-\mu) \alpha_{X A}}{\left[(1-\mu)\left(-2+3 \alpha_{X B}\right)\right]\left[(1-\mu)\left(-1+4 \alpha_{X C}\right)\right]}, \\
& \alpha_{X A} \in[0,1], \alpha_{X B} \in\left(\frac{2}{3}, 1\right], \alpha_{X C} \in\left[0, \frac{1}{4}\right) .
\end{aligned}
$$

In particular, for the level $\mu=0$ formula (64) takes the form:

$$
\begin{aligned}
& Z_{3}: z_{3}^{g r}=\frac{1+2 \alpha_{X A}}{\left[-2+3 \alpha_{X B}\right]\left[-1+4 \alpha_{X C}\right]}, \\
& \alpha_{X A} \in[0,1], \alpha_{X B} \in\left(\frac{2}{3}, 1\right], \alpha_{X C} \in\left[0, \frac{1}{4}\right) .
\end{aligned}
$$

This granule is visualized in Fig. 16. As can be seen, it is different from other granules. 
The last component result $Z_{4}$ of the division is also an information granule existing in the $5 \mathrm{D}$-space. For particular $\mu$-levels it has the form:

$$
\begin{aligned}
& Z_{4}=\frac{A}{B_{2} C_{2}}: \\
& z_{4}^{g r}=\frac{(1+\mu)+2(1-\mu) \alpha_{X A}}{\left[(1-\mu)\left(-2+3 \alpha_{X B}\right)\right]\left[(1-\mu)\left(-1+4 \alpha_{X C}\right)\right]}, \\
& \alpha_{X A} \in[0,1], \alpha_{X B} \in\left(\frac{2}{3}, 1\right], \alpha_{X C} \in\left(\frac{1}{4}, 1\right] .
\end{aligned}
$$

In particular, for the level $\mu=0$ the component granule can be simplified:

$$
\begin{aligned}
& Z_{4}: z_{4}^{g r}=\frac{1+2 \alpha_{X A}}{\left[-2+3 \alpha_{X B}\right]\left[-1+4 \alpha_{X C}\right]}, \\
& \alpha_{X A} \in[0,1], \alpha_{X B} \in\left(\frac{2}{3}, 1\right], \alpha_{X C} \in\left(\frac{1}{4}, 1\right] .
\end{aligned}
$$

This component granule is shown in Fig. 16 and it can be seen that it differs from other component granules. For other fractional $\mu$-levels, distances between component granules are different from granule distances for the level $\mu=0$. For the level $\mu=1$, the division result is undetermined. Because in the real system only one value of each variable $\alpha_{X A}, \alpha_{X B}, \alpha_{X C}$ is true, therefore also one division result $z=x_{A} /\left(x_{B} x_{C}\right)$ exists. This result is a single point that can lie only in one of the four component granules $Z_{1}-Z_{4}$.

4.4. Case 4. Now, let us examine the division case with a denominator in which the second-order polynomial occurs, (68). Value of the variable $x_{B}$ is uncertain and is expressed by the fuzzy value $X_{B}$ given by triple $(2,3,5)$. Fuzzy values $A$ and $X_{B}$ are shown in Fig. 17.

$$
Z=\frac{A}{B}=\frac{(1,2,4)}{X_{B}^{2}-10 X_{B}+21}, \quad X_{B}=(2,3,5)
$$

The denominator in formula (68) has two roots: $x_{B}=3$ and $x_{B}=7$. The fuzzy value $X_{B}$ contains the root $x_{B}=3$. The division result is indeterminate for this value, so MF of $X_{B}$ should be decomposed into two parts as shown in Fig. 18.
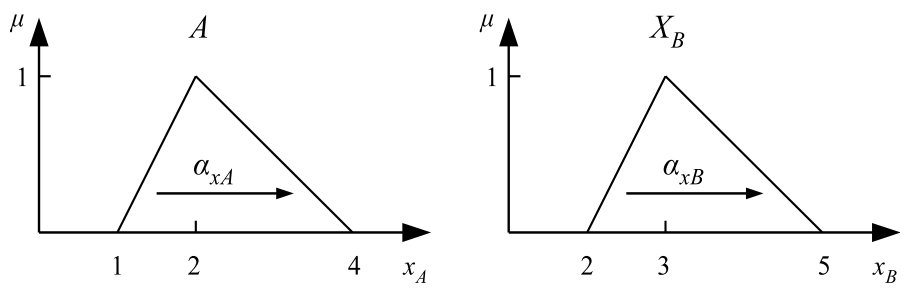

Fig. 17. Membership functions of fuzzy values $A$ and $X_{B}$ occurring in the division (68)

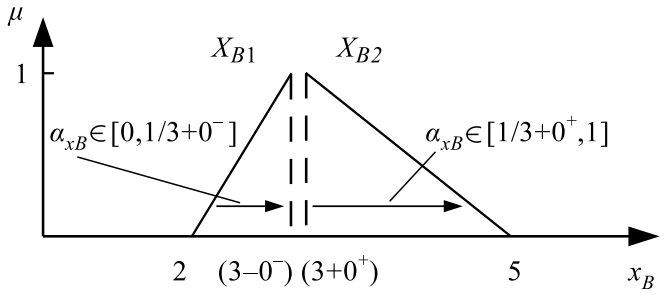

Fig. 18. Decomposition of membership function of fuzzy value $X_{B}$ into two parts, $x_{B} \neq 3, \alpha_{X B} \neq 1 / 3$

Formula (69) presents horizontal MFs of fuzzy values $A$, $X_{B 1}$ and $X_{B 2}$.

$$
\begin{aligned}
A: x_{A}=(1+\mu)+3(1-\mu) \alpha_{X A}, & \mu, \alpha_{X A} \in[0,1], \\
X_{B 1}: x_{B 1}=(2+\mu)+3(1-\mu) \alpha_{X B}, & \alpha_{X B} \in\left[0, \frac{1}{3}\right), \\
X_{B 2}: x_{B 2}=(2+\mu)+3(1-\mu) \alpha_{X B}, & \alpha_{X B} \in\left(\frac{1}{3}, 1\right]
\end{aligned}
$$

After inserting horizontal MFs into formula (68) we have:

$$
\begin{gathered}
Z_{1}: z_{1}^{g r}=\frac{(1+\mu)+3(1-\mu) \alpha_{X A}}{D}, \\
\mu, \alpha_{X A} \in[0,1], \alpha_{X B} \in\left[0, \frac{1}{3}\right), \\
Z_{2}: z_{2}^{g r}=\frac{(1+\mu)+3(1-\mu) \alpha_{X A}}{D}, \\
\mu, \alpha_{X A} \in[0,1], \alpha_{X B} \in\left(\frac{1}{3}, 1\right],
\end{gathered}
$$

where:

$D=\left[(2+\mu)+3(1-\mu) \alpha_{x A}\right]^{2}-10\left[(2+\mu)+3(1-\mu) \alpha_{x B}\right]+21$.

As can be seen from formula (70), the division result consists of two granules $Z_{1}$ and $Z_{2}$ which exist in 4D-space $z=f\left(\mu, \alpha_{X A}, \alpha_{X B}\right)$, hence they cannot be precisely visualized. However, they can be visualized in a simplified way in $2 \mathrm{D}$-space for particularly chosen membership levels $\mu$. For the level $\mu=0$ formula (70) takes the form:

$$
\begin{gathered}
Z_{1}: z_{1}^{g r}=\frac{1+3 \alpha_{X A}}{9 \alpha_{X B}^{2}-18 \alpha_{X B}+5}, \\
\mu, \alpha_{X A} \in[0,1], \alpha_{X B} \in\left[0, \frac{1}{3}\right), \\
Z_{2}: z_{2}^{g r}=\frac{(1+\mu)+3(1-\mu) \alpha_{X A}}{9 \alpha_{X B}^{2}-18 \alpha_{X B}+5}, \\
\mu, \alpha_{X A} \in[0,1], \alpha_{X B} \in\left(\frac{1}{3}, 1\right]
\end{gathered}
$$

Component granules $Z_{1}$ and $Z_{2}$ of the division result are shown in Fig. 19. 


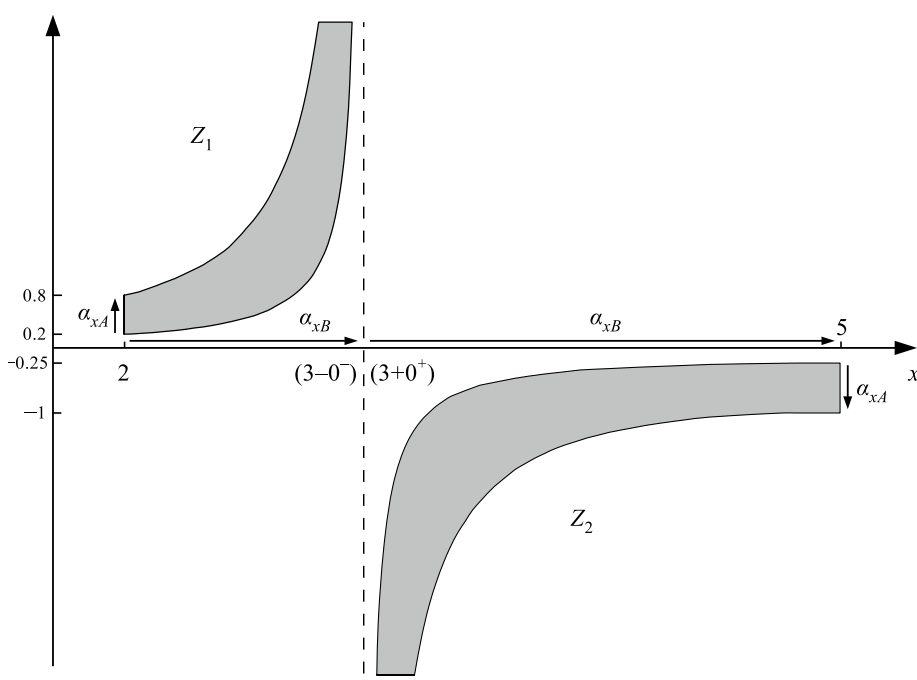

Fig. 19. Two component granules $Z_{1}$ and $Z_{2}$ of the division result determined by formula (71), $x_{B} \neq 3, \alpha_{X B} \neq 1 / 3, z \notin[-0.25,0.2]$

The true, crisp result of the division (70) lies in one of two granules: $Z_{1}$ or $Z_{2}$. In a real proble,m we sometimes have some additional knowledge, e.g. that the division result is negative. Then the true result can be located only in $Z_{2}$, which means decreasing the uncertainty.

4.5. Case 5. Solving linear equation systems is a frequent task in practical problems [26-28]. Coefficients occurring in the equations are often uncertain. A typical example is an economical plan model for the next period as e.g. balance model of Leontief for state economy [29]. Solving a linear equation system requires division by the system determinant $D$ which also is uncertain and can contain negative numbers, zero and positive numbers. Such situation results in multi-granular solutions. Let us consider equation system (72) with uncertain coefficients determined by triangle MFs.

$$
\begin{aligned}
& A_{1} x_{1}+B_{1} x_{2}=C_{1} \\
& A_{2} x_{1}+B_{2} x_{2}=C_{2}
\end{aligned}
$$

MFs of particular coefficients can be expressed in the form of triples: $A_{1}=(1,2,4), B_{1}=(3,5,6), C_{1}=(15,16,17)$, $A_{2}=(2,3,5), B_{2}=(7,8,9), C_{2}=(21,24,25)$, Fig. 20.

Horizontal MFs of uncertain coefficients can be determined as:

$$
\begin{aligned}
& A_{1}: x_{A 1}=(1+\mu)+3 \alpha_{A 1}(1-\mu), \\
& B_{1}: x_{B 1}=(3+2 \mu)+3 \alpha_{B 1}(1-\mu), \\
& C_{1}: x_{C 1}=(15+\mu)+2 \alpha_{C 1}(1-\mu), \\
& A_{2}: x_{A 2}=(2+\mu)+3 \alpha_{A 2}(1-\mu), \\
& B_{2}: x_{B 2}=(7+\mu)+2 \alpha_{B 2}(1-\mu), \\
& C_{2}: x_{C 2}=(21+3 \mu)+4 \alpha_{C 2}(1-\mu), \\
& \mu, \alpha_{A 1}, \alpha_{B 1}, \alpha_{C 1}, \alpha_{A 2}, \alpha_{B 2}, \alpha_{C 2} \in[0,1] .
\end{aligned}
$$
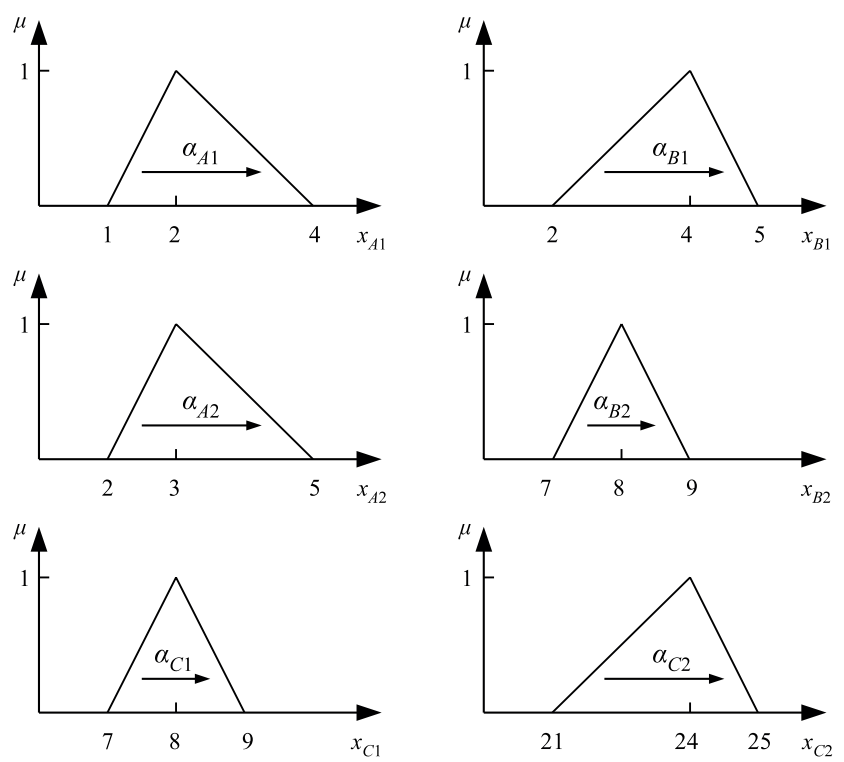

Fig. 20. Membership functions of uncertain coefficients $A_{1}, B_{1}, C_{1}, A_{2}$, $B_{2}, C_{2}$ occurring in the equation system (72)

The determinant $D$ of the equation system (72) has the form $D=x_{A 1} x_{B 2}-x_{A 2} x_{B 1}$ and solutions $z_{1}^{g r}$ and $z_{2}^{g r}$ can be formulated as:

$$
\begin{aligned}
x_{1}^{g r} & =\frac{x_{B 2} x_{C 1}-x_{B 1} x_{C 2}}{x_{A 1} x_{B 2}-x_{A 2} x_{B 1}}, \\
x_{2}^{g r} & =\frac{x_{A 1} x_{C 2}-x_{A 2} x_{C 1}}{x_{A 1} x_{B 2}-x_{A 2} x_{B 1}} .
\end{aligned}
$$

To check whether solutions $z_{1}^{g r}$ and $z_{2}^{g r}$ really have character of multi-granules it is sufficient to examine them for the membership level $\mu=0$. For this level solutions (74) take the form:

$$
\begin{aligned}
& x_{1}^{g r}= \frac{\left(7+2 \alpha_{B 2}\right)\left(15+2 \alpha_{C 1}\right)-}{\left(1+3 \alpha_{A 1}\right)\left(7+2 \alpha_{B 2}\right)-} \\
& \frac{-\left(3+3 \alpha_{B 1}\right)\left(21+4 \alpha_{C 2}\right)}{-\left(2+3 \alpha_{A 2}\right)\left(3+3 \alpha_{B 1}\right)}, \\
& x_{2}^{g r}= \frac{\left(1+3 \alpha_{A 1}\right)\left(21+4 \alpha_{C 2}\right)-}{\left(1+3 \alpha_{A 1}\right)\left(7+2 \alpha_{B 2}\right)-} \\
& \frac{-\left(2+3 \alpha_{A 2}\right)\left(15+2 \alpha_{C 1}\right)}{-\left(2+3 \alpha_{A 2}\right)\left(3+3 \alpha_{B 1}\right)}, \\
& \alpha_{A 1}, \alpha_{B 1}, \alpha_{C 1}, \alpha_{A 2}, \alpha_{B 2}, \alpha_{C 2} \in[0,1] .
\end{aligned}
$$

Let us notice that the nominator and denominator of $z_{1}^{g r}$ in (75) are not independent. They are coupled by RDM-variables $\alpha_{B 1}$ and $\alpha_{B 2}$. Similar coupling occurs in $z_{2}^{g r}$ by RDM-variables $\alpha_{A 1}$ and $\alpha_{A 2}$. To check whether solutions $z_{1}^{g r}$ and $z_{2}^{g r}$ have the form of multi-granules or not, the denominator $D$ of the equation system (75) for the level $\mu=0$ should be examined.

$D=\left(1+3 \alpha_{A 1}\right)\left(7+2 \alpha_{B 2}\right)-\left(2+3 \alpha_{A 2}\right)\left(3+3 \alpha_{B 1}\right)$,

$\alpha_{A 1}, \alpha_{B 1}, \alpha_{A 2}, \alpha_{B 2}, \in[0,1]$ 


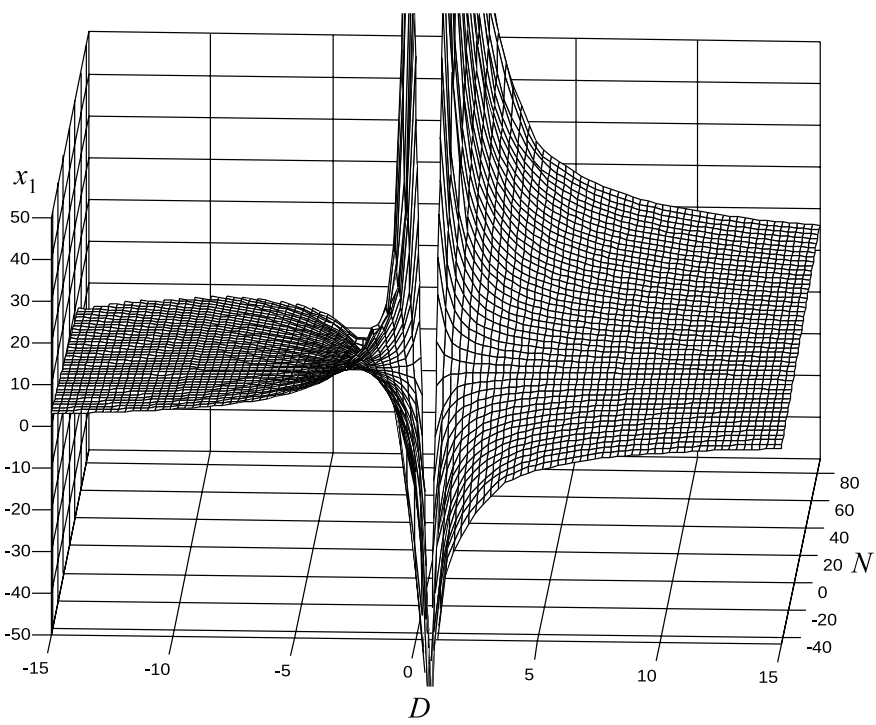

Fig. 21. Visualization of 2-granularity of $x_{1}^{g r}=x_{1,1}^{g r} \cup x_{1,2}^{g r}$ as the function of the denominator $D$ and the numerator $N$ of the solution (75)

Because the denominator $D$ is a monotonic function of RDM-variables, its extremes lie in corners of its domain $\alpha_{A 1} \times \alpha_{B 1} \times \alpha_{A 2} \times \alpha_{B 2}$. To detect these extremes, the denominator value for all combinations of RDM variables $\alpha_{A 1}, \alpha_{B 1}, \alpha_{A 2}, \alpha_{B 2}$ for values 0 and 1 must be calculated. Results of this examination are given in (77).

$$
\begin{aligned}
& \min D=D\left(\alpha_{A 1}=0, \alpha_{B 1}=1 \alpha_{A 2}=1, \alpha_{B 2}=0\right)=-23 \\
& \max D=D\left(\alpha_{A 1}=1, \alpha_{B 1}=0 \alpha_{A 2}=0, \alpha_{B 2}=1\right)=30
\end{aligned}
$$

It can be seen that $D \in[-23,30]$. Occurrence of zero in the interval of the denominator $D$ means that the solution of the equation system is two-granular: $x_{1}^{g r}=x_{1,1}^{g r} \cup x_{1,2}^{g r}$ and $x_{2}^{g r}=x_{2,1}^{g r} \cup x_{2,2}^{g r}$.

$$
\begin{aligned}
x_{1,1}^{g r}= & f_{1,1}\left(\alpha_{A 1}, \alpha_{B 1}, \alpha_{C 1}, \alpha_{A 2}, \alpha_{B 2}, \alpha_{C 2}\right), \\
& \text { for } D\left(\alpha_{A 1}, \alpha_{B 1}, \alpha_{A 2}, \alpha_{B 2}\right) \in[-23,0) \\
x_{1,2}^{g r}= & f_{1,2}\left(\alpha_{A 1}, \alpha_{B 1}, \alpha_{C 1}, \alpha_{A 2}, \alpha_{B 2}, \alpha_{C 2}\right), \\
& \text { for } D\left(\alpha_{A 1}, \alpha_{B 1}, \alpha_{A 2}, \alpha_{B 2}\right) \in(0,30]
\end{aligned}
$$

The 3D-projection from the 7D-space of the two-granular solution $z_{1}^{g r}$ is shown in Fig. 21. Projection of the solution $x_{2}^{g r}$ is of similar character (Fig. 22).

In Figs. 21 and 22 one can distinctly see the separation of component-solution granules. One can also see that component granules $x_{1,1}^{g r}$ and $x_{1,2}^{g r}$ are different functions.

When the solution of the equation system (72) is presented in a too low-dimensional or inappropriate space (as in Fig. 23), the separation of solution granules is not visible. However, such inappropriate presentation can be found in many papers concerning uncertain equation systems, e.g in [26].

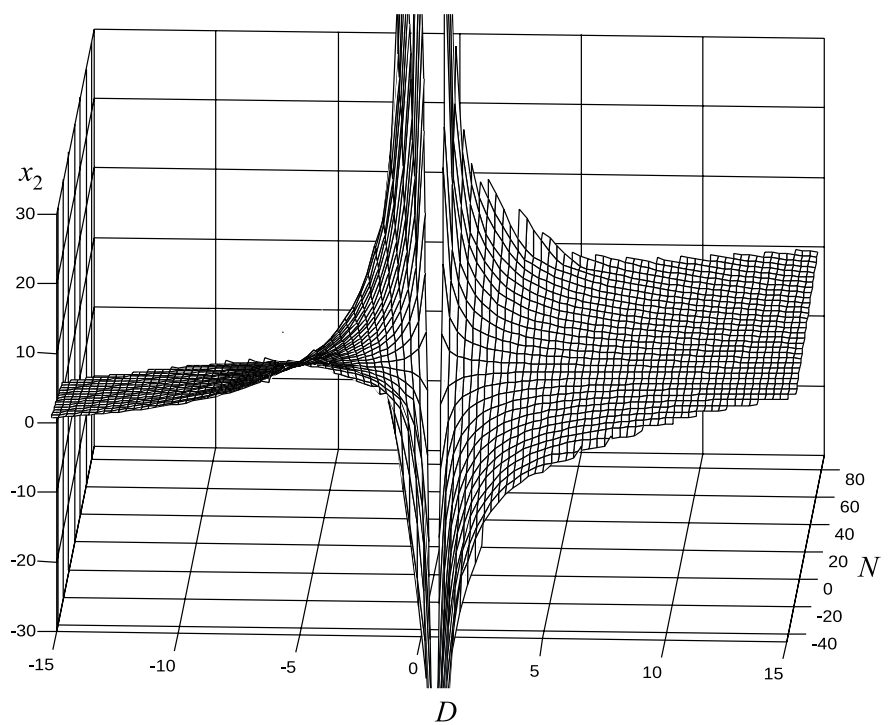

Fig. 22. Visualization of $x_{2}^{g r}=x_{2,1}^{g r} \cup x_{2,2}^{g r}$ as the function of the denominator $D$ and the numerator $N$ of the solution (75)

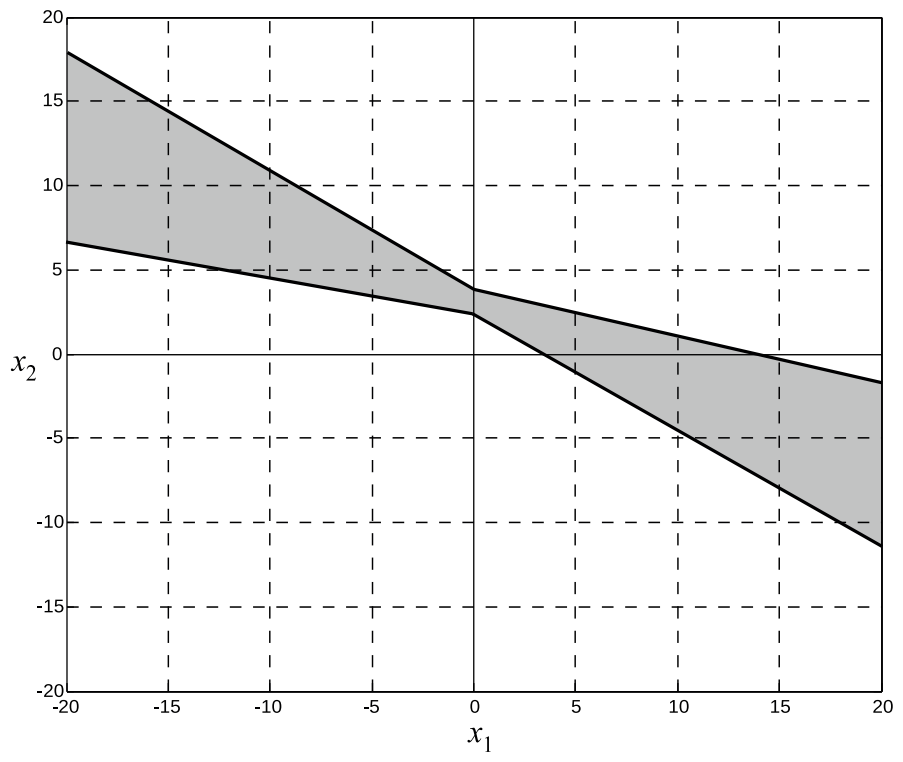

Fig. 23. Visualization of the set of possible solutions (75) for $\alpha_{A 1}$, $\alpha_{B 1}, \alpha_{C 1}, \alpha_{A 2}, \alpha_{B 2}, \alpha_{C 2} \in[0,1]$ in the space $X_{1} \times X_{2}$; no separation is visible

\section{Conclusions}

Solving problems described by uncertain mathematical models which explicitly or sometimes implicitly (like fuzzy equation systems) contain the division operation can provide multi-granular, not compact solutions consisting of two or more component granules which are distinctly separated. Sometimes, the granule separation is considerable, as has been shown in examples. The multi-granularity hinders problem solving, especially when the multi-granular solution of one sub-model has to be introduced in the next submodel in which division 
also occurs. Fuzzy RDM arithmetic allows for detecting the multi-granularity and allows for taking into account possible couplings (correlations) existing between particular problem variables, as in the case of fuzzy system equations where such couplings exists between the numerator and the denominator of system solutions. Being aware of the multi-granularity of the solutions is very important for correct and precise solving of uncertain problems.

\section{REFERENCES}

[1] D. Dubois and H. Prade, "Operations on fuzzy numbers", International Journal of Systems Science 9 (6), 613-626 (1978).

[2] P. Dutta, H. Boruah, and T. Ali, "Fuzzy Arithmetic with and without using $\alpha$-cut method: A Comparative study", International Journal of Latest Trends in Computing 2 (1), 99-107 (2011).

[3] J. Fodor and B. Bede, "Arithmetics with fuzzy numbers: A comparative overview", Proc. Slovakian-Hungarian Joint Symposium on Applied Machine Intelligence, 54-68 (2006).

[4] W. Pedrycz, A. Skowron, and V. Kreinovich, Handbook of Granular Computing, John Wiley \& Sons, Chichester, 2008.

[5] A. Piegat and M. Landowski, "Two interpretations of multidimensional RDM interval arithmetic: Multiplication and division”, International Journal of Fuzzy Systems 15 (4), 486-496 (2013).

[6] A. Piegat and M. Pluciński, "Fuzzy number addition with the application of horizontal membership functions", The Scientific World Journal 2015, Article ID 367214 (2015).

[7] K. Tomaszewska, "The application of horizontal membership function to fuzzy arithmetic operations", Journal of Theoretical and Applied Computer Science 8 (2), 3-10 (2014).

[8] R.E. Moore, R.B. Kearfott, and M.J. Cloud, Introduction to Interval Analysis, Society for Industrial and Applied Mathematics, Philadelphia, 2009.

[9] B. Liu, Uncertainty Theory, Springer Verlag, Berlin, 2010.

[10] Q.X. Li and S.F. Liu, "The foundation of the grey matrix and the grey input-output analysis", Applied Mathematical Modelling 32 (3), 267-291 (2008).

[11] A. Piegat and M. Pluciński, "Computing with words with the use of inverse RDM models of membership functions", International Journal of Applied Mathematics and Computer Science 25 (3), 675-688 (2015)

[12] L.A. Zadeh, "Fuzzy logic = computing with words", IEEE Transactions on Fuzzy Systems 4 (2), 103-111 (1996).
[13] K. Tomaszewska and A. Piegat, "Application of the horizontal membership function to the uncertain displacement calculation of a composite massless rod under a tensile load", in Soft Computing in Computer and Information Science, pp. 63-72, eds. A. Wiliński, I. El Fray and J. Pejaś, Springer, 2015.

[14] J. Smoczek, "Interval arithmetic-based fuzzy discrete-time crane control scheme design", Bull. Pol. Ac.: Tech 61 (4), 863-870 (2013).

[15] A. Niewiadomski and M. Kacprowicz, "Higher order fuzzy logic in controlling selective catalytic reduction systems", Bull. Pol. Ac.: Tech 62 (4), 743-750 (2014).

[16] J. Smoczek, "P1-TS fuzzy scheduling control system design using local pole placement and interval analysis", Bull. Pol. Ac.: Tech 62 (3), 455-464 (2014).

[17] T. Chen, "Remarks on the subtraction and division operations over intuitionistic fuzzy sets and interval-valued fuzzy sets", International Journal of Fuzzy Systems 9 (3), 169-173 (2007).

[18] A. Piegat and M. Landowski, "Aggregation of inconsistent expert opinions with use of horizontal intuitionistic membership functions", in Novel Developments in Uncertainty Representation and Processing, pp. 215-224, eds. K. Atanassov, O. Castillo, and J. Kacprzyk, Springer, 2016.

[19] B. Qing and H. Wong, "Generalized interval-valued fuzzy variable precision rough sets", International Journal of Fuzzy Systems 16 (4), 554-565 (2014)

[20] A. Kaufmann and M.M. Gupta, Introduction to Fuzzy Arithmetic, Van Nostrand Reinhold, New York, 1991.

[21] A. Piegat, Fuzzy Modeling and Control, Physica Verlag, Heidelberg, 2001.

[22] M. Hanss, Applied Fuzzy Arithmetic, Springer Verlag, Berlin, 2005.

[23] M. Hanss, "The transformation method for the simulation and analysis of systems with uncertain parameters", Fuzzy Sets and Systems 130 (2), 277-289 (2002).

[24] G.J. Klir and Y. Pan, "Constrained fuzzy arithmetic: Basic questions and some answers", Soft Computing 2 (2), 100-108 (1998).

[25] A. Piegat and M. Landowski, "Horizontal membership function and examples of its applications", International Journal of Fuzzy Systems 17 (1), 22-30 (2015).

[26] S.P. Shary, "On optimal solution of interval linear equations", SIAM Journal on Numerical Analysis 32 (2), 610-630 (1995).

[27] P. Sevastjanov and L. Dymova, "A new method for solving interval and fuzzy equations: Linear case", Information Sciences 179 (7), 925-937 (2009).

[28] L. Dymova, Soft Computing in Economics and Finance, Springer Verlag, Berlin, 2011.

[29] W.W. Leontief, Input-Output Economics, Oxford University Press on Demand, 1986. 